\title{
Vacuum decay in quantum field theory
}

\author{
Esteban Calzetta \\ Departamento de Física, Universidad de Buenos Aires, Ciudad Universitaria, 1428 Buenos Aires, Argentina
}

\author{
Albert Roura and Enric Verdaguer* \\ Departament de Física Fonamental, Universitat de Barcelona, Av. Diagonal 647, 08028 Barcelona, Spain
}

(Received 11 June 2001; published 16 October 2001)

\begin{abstract}
We study the contribution to vacuum decay in field theory due to the interaction between the long- and short-wavelength modes of the field. The field model considered consists of a scalar field of mass $M$ with a cubic term in the potential. The dynamics of the long-wavelength modes becomes diffusive in this interaction. The diffusive behavior is described by the reduced Wigner function that characterizes the state of the longwavelength modes. This function is obtained from the whole Wigner function by integration of the degrees of freedom of the short-wavelength modes. The dynamical equation for the reduced Wigner function becomes a kind of Fokker-Planck equation which is solved with suitable boundary conditions enforcing an initial metastable vacuum state trapped in the potential well. As a result a finite activation rate is found, even at zero temperature, for the formation of true vacuum bubbles of size $M^{-1}$. This effect makes a substantial contribution to the total decay rate.
\end{abstract}

DOI: 10.1103/PhysRevD.64.105008

PACS number(s): 03.70.+k, 05.40.Ca, 11.10.Ef

\section{INTRODUCTION}

In this paper we report our preliminary findings within a larger program which aims at the development of a theory of nonequilibrium first order phase transitions, such as have occurred in the Early Universe (grand unified and electroweak symmetry breaking [1]) and, possibly, in the first stages of heavy ion collisions (chiral symmetry breaking and confinement $[2,3])$. For this reason, we must seek a description of the decay process which emphasizes the dynamical aspects over the static aspects encoded in the effective potential.

Vacuum decay in field theory is described with a potential which displays a local minimum, separated from the absolute minimum by a potential barrier. A system prepared in the false vacuum state (metastable phase) within the potential well may decay in essentially two different ways: namely (a) by tunneling effect, that is, by going through the barrier in a classically forbidden trajectory [4-6], or else, (b) by activation, that is, by jumping above the barrier $[7,8]$. In either case, the decay probability follows the law $P \sim A \exp (-B)$ which gives the probability per unit time and unit volume to nucleate a region of the stable phase within the metastable phase. In the tunneling effect, $B=S / \hbar$, where $\hbar$ is Planck's constant and $S$ is the action for the trajectory which goes under the barrier in imaginary time [9]. In thermal activation, $B=V_{s} /\left(k_{B} T\right)$, where $k_{B}$ is Boltzmann's constant, $T$ the temperature, and $V_{s}$ is the height of the free energy measured from the false vacuum [10-12]. Thus, activation disappears as $T \rightarrow 0$.

In systems with few degrees of freedom, there must be an external agent, typically a thermal source, for activation to be possible. Our thesis is that in field theories there is a phenomenon similar to activation even at zero temperature. This comes from the observation that in a field theory, when a

\footnotetext{
*Also at Institut de Física d'Altes Energies (IFAE), Barcelona, Spain.
}

mode decomposition of the field is made, there are only a few long wavelengths modes which are unstable and decay. These nearly homogeneous modes may be regarded as an open system, which interacts with the environment provided by the shorter wavelength modes. It is then possible to describe the quantum evolution of the system in terms of an effective dynamics, whereby the interaction with the environment results in the onset of dissipation and noise.

The ultimate reason for the presence of a finite activation rate even at zero temperature is that, for a generic field theory the dynamics of these homogeneous modes is anharmonic enough to contain Fourier components with frequencies above the threshold for excitation of the short wavelength modes. This results in an energy transfer from the long wavelength or homogeneous modes to the short wavelength or inhomogeneous modes through particle creation. As demanded by the energy balance, and encoded in the fluctuation-dissipation theorem, this energy flow is compensated by a stochastic force on the homogeneous modes, originated in fluctuations of the inhomogeneous modes. Thus the dynamics of the homogeneous modes becomes diffusive, even if, properly speaking, there is no external "environment" to the field [13].

We wish to stress that this is not only a theoretical possibility. In this paper, we will show through a detailed analysis of a concrete model that the activation rate makes a substantial contribution to the full decay rate even at zero temperature. In the process, we shall develop the necessary formalism to compute the activation rate to leading order in $\hbar$.

The key ingredient will be the description of the quantum state of the long wavelength modes of the field by means of the reduced Wigner function. This function has the same information as the reduced density matrix of an open quantum system but is similar in many aspects to a distribution function in phase-space. The dynamical equation for the reduced Wigner function (master equation) includes noise terms produced by the short wavelength modes to quadratic 
order in the parameter coupling the short and long wavelength modes. We shall derive the tunneling rate from an analysis of the decay of nontrivial solutions of the master equation, after suitable boundary conditions have been enforced.

The master equation contains all required information on vacuum decay, including both the "tunneling" aspect of the homogeneous mode as well as "activation," i.e. the effect due to the backreaction of the inhomogeneous modes, but in this paper we concentrate on the activation aspect. Thus, whereas the instanton method [9], for instance, seems to be best suited to describe the pure tunneling of the homogeneous mode, the effect due to the backreaction of the inhomogeneous modes seems to be best described by the present method. Another advantage of this approach, which was one of our first motivations, is that whereas the instanton method works well for processes not far from equilibrium, this method should work also for out of equilibrium situations since it is based on a real time evolution equation such as the master equation.

Our conclusion shall be that, for a physically well motivated system-environment separation, the contribution to the exponent coming from quantum fluctuations in the short wavelength modes is comparable to, but distinguishable from, the contribution from tunneling under the barrier by the long wavelength modes themselves. Which contribution is actually dominant will depend on the specifics of each model. Therefore, the approaches that underplay backreaction from the short wavelength modes, underestimate the tunneling rate, and may even miss the largest contribution.

Comparing with the literature on tunneling in open and driven systems, the main difference is that we do not assume a priori any features of the noise and dissipation, but rather derive them from the underlying unitary field theory. In practice, this means that we shall have to deal with non-local dissipation and colored noise. Also for simplicity, we shall focus on computing the exponent in the tunneling rate to leading order in $\hbar$, and at zero temperature. As a matter of fact, this is the difficult case, since at high temperature the interaction of the short and long wavelength modes is just another aspect of the interaction with the heat bath.

The paper is organized as follows. In Sec. II we briefly review current theories of vacuum decay and place our work in that context. In Sec. III we present the basic derivation of the master equation for the reduced Wigner function for a quantum Brownian motion (QBM) model which is of relevance for our problem. The model is an open quantum system consisting of a massive particle in an arbitrary potential coupled to an infinite set of harmonic oscillators, the coupling is linear in the system variables but quadratic in the oscillator coordinates. For our problem the master equation reduces to a Fokker-Planck-like equation. In Sec. IV we consider a field theory model consisting of a massive scalar field with a negative cubic potential term. The long and short wavelength modes of the field are separated and we reduce the problem to a system-environment interaction similar to the QBM model discussed in the previous section. The relevant kernels for the corresponding Fokker-Planck equation are calculated. Section V contains the derivation of the tun- neling rate from the analysis of the solutions to the FokkerPlanck equation. We discuss our results in the final section. A number of Appendixes contain some of the unavoidable technical details.

\section{VACUUM DECAY: A SURVEY}

\section{A. Vacuum decay in systems with one degree of freedom}

The twin issues of thermal activation and spontaneous nucleation have a long and distinguished history; see Refs. $[14,10]$ for a review. In its simplest formulation, we deal with a quantum mechanical system with one degree of freedom $x$ and Hamiltonian $H=p^{2} / 2+V(x)$, where $V$ has the generic form shown in the figure. It is an experimental fact that even if we prepare the system to be confined to the neighborhood of the "false vacuum" $x \sim 0$, and the barrier is much higher than the typical energies accessible to the system $\left[E \sim \hbar V^{\prime \prime}(0)\right.$ in the quantum mechanical problem, $E$ $\sim k T$ at finite temperature], the system will find a way to escape from the potential well after some typical time $\tau$ has elapsed. The problem is to estimate the "mean life" $\tau$ or equivalently the "tunneling rate" $\tau^{-1}$.

Vacuum decay can be formulated as a steady state problem if we inject particles into the system in order to keep a constant population in the false vacuum state. We then have a constant flux of particles impinging on the barrier from the left, and the problem reduces to the computation of the transmission coefficient [4]. In quantum mechanics, this is readily obtained within the WKB approximation, and the result is the Arrhenius-like expression

$$
\tau^{-1} \sim \Delta \exp (-S / \hbar), \quad S=\int_{0}^{x_{e x i t}} d x \sqrt{2 V(x)},
$$

where $\Delta$ is a prefactor of order 1 , and we have set the classical energy of the false vacuum to 0 .

This formula describes vacuum decay through tunneling, that is, an essentially quantum phenomenon. If we allow the system to interact with an external noise source (typically a heat bath at a given temperature $T$ ), then the energy of the system alone is no longer conserved, and the system can jump over the barrier, resulting in vacuum decay through activation. The activation rate has been computed, within the "constant flux" approach, by Kramers [7] and Langer [8]. They show that Eq. (1) still holds, but the exponent $S / \hbar$ becomes $F_{s} / k T$, where $F_{s}$ is the activation free energy, i.e. the height of the free energy barrier to be overcome.

A less artificial approach attempts to compute the actual time evolution of the false vacuum state $\Psi_{F}$. This is not a stationary state of the system, but it may be expanded in energy eigenstates $\psi_{E}$. The amplitude of $\psi_{E}$ in the expansion of $\Psi_{F}$ peaks around $E \sim 0$, and for small energy may be approximated by the Breit-Wigner form $[5,6]$

$$
\Psi_{F}(x, t) \sim \int d E e^{-i E t / \hbar} \frac{\psi_{E}(x)}{E^{2}+\lambda^{2}} .
$$


At long, but not too long, times we obtain $\Psi_{F} \sim C e^{-\lambda t / \hbar}$. The false vacuum behaves as an energy eigenstate with complex energy $E_{F} \sim-i \lambda$ (complex energies and eigenstates may be defined within an extended formalism of quantum mechanics [15]), and the mean life is $\tau \sim \hbar \lambda^{-1}$.

The calculation of activation rates in the "complex energy" approach has been worked out by Langer [16]. The idea is to define a "free energy" for an ensemble of unstable configurations including the critical droplet. This "free energy" is complex, and its imaginary part is related to the mean life as in the quantum mechanical problem. The physical free energy, of course, is real, and it is given by Maxwell's construction [17].

Coleman and collaborators have proposed a simple and elegant way to compute the complex false vacuum energy [9]. The idea is that the vacuum energy can be expressed in terms of a path integral over Euclidean histories with appropriate boundary conditions. For unstable systems, the path integral must be computed by analytic continuation, and an imaginary part appears. In certain cases it is possible to show that the path integral is dominated by the contributions from a discrete set of saddle points, corresponding to sequences of "bounces" against the inner sides of the barrier with little or no overlap between bounces. Then the formula in Eq. (1) is recovered, where $S$ is now interpreted as the Euclidean action for the one bounce solution, also called the "instanton."

The instanton method is easily generalized to the thermal case [11]. The idea is to write the partition function for the unstable system as a path integral over Euclidean configurations with periodicity $\beta \hbar \quad(\beta=1 / k T)$ in Euclidean time, and then to evaluate the path integral in the saddle point approximation. Due to new boundary conditions, the thermal instanton may not be the same as the instanton at $T=0$. The change in the nature of the instanton gives a simple and compelling interpretation of the crossover from spontaneous transition to thermal activation [12].

The tunneling rate can also be derived from the large order behavior of perturbation theory [18].

\section{B. Tunneling in systems with few degrees of freedom}

All approaches discussed so far have natural generalizations to systems with few degrees of freedom. In the case of the instanton approach, the generalization is almost immediate, only one has to take care of symmetries of the system which may appear as zero modes in the spectrum of perturbations around the instanton solution, thus causing an apparent divergence of the path integral. These symmetries may be handled by isolating them as collective modes prior to the saddle point evaluation of the path integral [19].

The "constant flux" approach is implemented by seeking a solution to the Schrödinger equation within the WKB or Born-Oppenheimer approximation [20]. The idea is to identify a single variable $x$ which parametrizes the "most probable scape path," namely the path across the saddle separating the false and true vacua. Then one uses a mixed ansatz for the wave function, whereby it is assumed to be Gaussian on all other variables, and of WKB form with respect to $x$. "Under the barrier," the WKB approximation leads to a
Hamilton-Jacobi equation for a particle moving in an inverted potential, the same dynamical problem one confronts in the instanton method, although now the potential may be modified by terms arising from the zero point energies of the transversal modes; but not of $x$ itself [21]. This solution is then matched to oscillatory solutions on either side of the barrier.

Conceptually, the WKB method has some advantages over the instanton method [22]. In the first place, the connection to the Schrödinger equation is much more straightforward. Also, one has more control of the quantum state of the transversal degrees of freedom, which then allows one to ask questions like whether tunneling is associated to particle creation [23-25]. Finally, it has proved easier to define the range of validity of the approximations involved in the WKB framework than in the Euclidean path integral one. However, they are likely to be equivalent in last analysis, and the conclusion that these methods work best when the tunneling variable $x$ is slow compared with the transversal modes probably holds equally well for both approaches [26]. We refer the reader to Ref. [27] for recent developments along these lines, and to Ref. [28] for an example of similar techniques in a different context.

Some nonequilibrium aspects of vacuum decay have been the focus of work by Boyanovsky et al. [29]. They show that realistic initial conditions usually imply a nonzero probability for the system to be at the unstable configuration in the saddle of the free energy surface. Starting from this configuration, the roll down of the system towards the true vacuum may be analyzed by usual nonequilibrium field theory methods. However, the initial amplitude is still computed by conventional methods, such as discussed so far.

\section{Tunneling in quantum field theory}

Gervais, Sakita and De Vega have applied the WKB method to tunneling in quantum field theory [30]; see Refs. $[31,32]$ for instanton and complex-time methods. In spite of the obvious similarities, there are some important differences between the problem of tunneling in field theory and in systems with few degrees of freedom. Some of these differences are technical in nature, such as the need to carefully account for loop corrections to the effective potential [33], and to adopt a regularization procedure to compute the prefactor in the Arrhenius formula [34-36], which would be divergent if naively computed. There is also a fundamental conceptual difference which we now discuss.

A field theory only makes sense, from the physical point of view, if it is understood as an effective theory describing the low frequency sector of a more fundamental theory, whose high frequency degrees of freedom may be totally unlike continuous fields; such as strings in elementary particle physics, discrete lattices in condensed matter applications, and molecules in hydrodynamics [37].

One clear way to bring this point home is by explicitly integrating out all modes with wave number $k>\Lambda$, where $\Lambda$ is some cutoff. The remaining modes are described by a coarse grained effective potential [38-43]. As $\Lambda$ decreases from $\infty$ to 0 , the coarse grained effective potential interpo- 
lates between the "bare" potential shown in the figure to the Maxwell construction free energy [44], showing the drastic effect of the short wavelength modes or high frequency sector on the physics of the long wavelength modes or low frequency sector. At some point the barrier separating the different metastable points disappears, reflecting the effect of averaging the field over distances much larger than a domain.

\section{Tunneling in open systems}

As we have seen from earlier studies of the coarse grained effective potential, tunneling in field theory should be properly posed as an open quantum system problem. However, the nature of tunneling in an open system cannot be described adequately by just computing changes in an effective potential. Besides the static changes reflected by the scale dependent effective potential, the dynamics of the long wavelength modes will become both dissipative and stochastic. The onset of dissipation and noise is also generic to semiclassical [13] and effective theories [45].

For the present discussion it is essential to realize that noise and dissipation are actually two aspects of a single phenomenon, the dynamical action and back-reaction between "system" and "environment." In equilibrium situations, this inner relationship can be made explicit through the "fluctuation-dissipation" relation [46].

A simple way to deal with tunneling in open systems is to model the environment explicitly within a larger systemenvironment complex, in effect reducing the problem to tunneling in many degrees of freedom [47-49]. However, it is essential to avoid approximations (such as assuming that the environment degrees of freedom perform linear oscillations around a prescribed trajectory of the system) which in practice underplay the backreaction of the environment on the system, and thus break the balance between fluctuation and dissipation. The relevance of the fluctuation-dissipation relation to tunneling has been emphasized in Ref. [50].

The broadening of the reduced Wigner function of the open system by external noise has been discussed in Ref. [51]. Unlike the present work, these authors consider an external noise source, whose spectral features may be chosen at will. In order to give an adequate account of backreaction, Bruinsma and Bak [52] have proposed treating the system as propagating in a random medium, the randomness being associated to the environmental variables. In a second step, a path integration over histories of the bath allows the computation of the tunneling rate. As in the present work, a serious consideration of backreaction leads to describing the system as a driven system, subject to stochastic forces originating from the environment.

The theory of vacuum decay in open systems has points of contact with the problem of decay in driven systems $[53,54]$, although in these later studies usually the properties of the driving force are assumed a priori, rather than derived from a more comprehensive model. It is also possible to obtain a path integral representation of the solution of a Langevin equation, whereby an open system may be subsumed into a larger field theory [55,56]. See Ref. [57] for further developments.

\section{E. Tunneling and the semiclassical approximation}

Semiclassical field theories, where some fields are treated as c-number, while the rest are described quantum mechanically, may be seen as open systems, with the classical fields as the system and the quantum fields as the environment. Quantum fluctuations in the system are registered as noise by the environment, and may induce transitions.

An early application of these ideas appeared within Starobinsky's "stochastic inflation" program [58]. Here the superhorizon modes of the inflaton field during inflation are the system whereas all other shorter wavelength modes are the environment; for a discussion of the validity of the semiclassical approximation, see [59,60]. Cosmological redshift causes a continuous streaming of modes from the environment to the system, which may be regarded as a white noise source. This noise may allow the system to hop over potential barriers, seeking the absolute minima. Eventually, a steady state distribution of cosmological domains is reached, not unlike that predicted by the Hartle and Hawking "wave function of the Universe" [22,61].

Much more generally, within the semiclassical approximation the backreaction to cosmological particle creation processes always has a stochastic component [13], for which reason the correct semiclassical description of the Early Universe ought to be formulated in terms of a stochastic "Einstein-Langevin" equation [62]. If we consider an ensemble of Universes, then we may introduce a distribution function obeying a Fokker-Planck-like equation [63]. This equation describes activation phenomena, which are the semiclassical version of Vilenkin's "creation from nothing" scenario [64].

\section{OPEN SYSTEMS AND THE REDUCED WIGNER FUNCTION}

Before dealing with field theory we will consider in this section a quantum Brownian motion (QBM) model which is typically used as a paradigm of an open quantum system. The system has an arbitrary potential and the coupling between the system and the environment is linear in the system variables but quadratic in the environment variables. That feature will be of relevance when dealing in Sec. IV with our field-theory model. The main result of this section is the derivation of the master equation for the reduced Wigner function of the QBM model to leading order in $\hbar$. This equation turns out to be a Fokker-Planck equation which is similar to that used by Kramers [7] to study the activation problem in statistical physics.

\section{A. A QBM model}

As our QBM model we consider a system consisting of a particle of unit mass $(M=1)$ described with a variable $x$ and subject to an arbitrary potential with a quadratic part corresponding to an oscillator of frequency $\Omega_{0}$ and an anharmonic part $V^{(n l)}(x)$, i.e. $V(x)=(1 / 2) \Omega_{0}^{2} x^{2}+V^{(n l)}(x)$, which is coupled to an environment consisting of an infinite set of harmonic oscillators with coordinates $q_{j}$. The action for the whole set of degrees of freedom is defined by 


$$
S\left[x,\left\{q_{j}\right\}\right]=S[x]+S\left[\left\{q_{j}\right\}\right]+S_{i n t}\left[x,\left\{q_{j}\right\}\right],
$$

where the system, environment and interaction actions are given, respectively, by

$$
\begin{gathered}
S[x]=\int d t\left(\frac{1}{2} \dot{x}^{2}-V(x)\right), \\
S\left[\left\{q_{j}\right\}\right]=\sum_{j} \int d t\left(\frac{1}{2} m \dot{q}_{j}^{2}-\frac{1}{2} m \omega_{j}^{2} q_{j}^{2}\right), \\
S_{\text {int }}\left[x,\left\{q_{j}\right\}\right]=\sum_{j} g \int d t x q_{j}^{2},
\end{gathered}
$$

where $g$ is a coupling constant, and we have assumed that the coupling is linear in the system variable but quadratic in the environment variables. The environment oscillators have all the same mass $m$ and their frequencies are $\omega_{j}$. At this point the potential $V^{(n l)}(x)$ is arbitrary, but later we will take a cubic potential, $V^{(n l)}(x)=-(\lambda / 6) x^{3}$, in this way the total potential will present a local minimum and a barrier as required to represent the system in a metastable phase. Also the parameters $\lambda$ and $g$ are unrelated, however when we consider the application to a field theory these parameters will coincide.

The reduced density matrix for our open quantum system at a certain final time $t_{f}$ is defined from the density matrix $\rho$ of the whole system by tracing out the environment degrees of freedom at that time

$$
\rho_{r}\left(x_{f}, x_{f}^{\prime}, t_{f}\right)=\int \prod_{j} d q_{j} \rho\left(x_{f},\left\{q_{j}\right\}, x_{f}^{\prime},\left\{q_{j}\right\}, t_{f}\right) .
$$

The reduced density matrix at time $t_{f}$ can be written in terms of the reduced density matrix at the initial time $t_{i}$ by the evolution equation,

$$
\rho_{r}\left(x_{f}, x_{f}^{\prime}, t_{f}\right)=\int d x_{i} d x_{i}^{\prime} J\left(x_{f}, x_{f}^{\prime}, t_{f} ; x_{i}, x_{i}^{\prime}, t_{i}\right) \rho_{r}\left(x_{i}, x_{i}^{\prime}, t_{i}\right),
$$

in terms of the propagator $J$, whose path integral representation is

$$
\begin{aligned}
J\left(x_{f}, x_{f}^{\prime}, t_{f} ; x_{i}, x_{i}^{\prime}, t_{i}\right)= & \int_{x_{i}}^{x_{f}} \mathcal{D} x \int_{x_{i}^{\prime}}^{x_{f}^{\prime}} \mathcal{D} x^{\prime} \exp _{\bar{\hbar}}^{i}\left(S[x]-S\left[x^{\prime}\right]\right. \\
& \left.+S_{I F}\left[x, x^{\prime}\right]\right)
\end{aligned}
$$

where $x_{i}=x\left(t_{i}\right), x_{f}=x\left(t_{f}\right)$ and similarly for the primed quantities, and $S_{I F}\left[x, x^{\prime}\right]$ is the Feynman and Vernon influence action [65]. When the system and the environment are initially uncorrelated the initial density matrix factorizes, i.e. $\rho\left(t_{i}\right)=\rho_{r}\left(t_{i}\right) \rho_{e}\left(t_{i}\right)$ (here $\rho_{e}$ stands for the environment density matrix); the influence functional, which is defined by $F\left[x, x^{\prime}\right]=\exp \left(i S_{I F}\left[x, x^{\prime}\right] / \hbar\right)$, can be expressed by

$$
\begin{aligned}
F\left[x, x^{\prime}\right]= & \prod_{j} \int d q_{j}^{(f)} d q_{j}^{(i)} d q_{j}^{\prime(i)} \int_{q_{j}^{(i)}}^{q_{j}^{(f)}} \mathcal{D} q_{j} \int_{q_{j}^{\prime(i)}}^{q_{j}^{(f)}} \mathcal{D} q_{j}^{\prime} \\
& \times \exp _{\bar{\hbar}}^{i}\left(S\left[\left\{q_{j}\right\}\right]-S\left[\left\{q_{j}^{\prime}\right\}\right]+S_{i n t}\left[x,\left\{q_{j}\right\}\right]\right. \\
& \left.-S_{\text {int }}\left[x^{\prime},\left\{q_{j}^{\prime}\right\}\right]\right) \rho_{e}\left(\left\{q_{j}^{(i)}\right\},\left\{q_{j}^{\prime(i)}\right\}, t_{i}\right),
\end{aligned}
$$

where $q_{j}^{(i)}=q_{j}\left(t_{i}\right), q_{j}^{\prime(i)}=q_{j}^{\prime}\left(t_{i}\right)$, and at the final times $q_{j}\left(t_{f}\right)=q_{j}^{(f)}=q_{j}^{\prime}\left(t_{f}\right)$.

Assuming a Gaussian initial state for the environment, $\rho_{e}$ is Gaussian and the influence functional can be computed perturbatively in $g$ from the path integral. Up to second order in $g[65]$ we have for the influence action,

$$
\begin{aligned}
S_{I F}\left[x, x^{\prime}\right]= & -2 \int_{t_{i}}^{t_{f}} d t \int_{t_{i}}^{t} d t^{\prime} \Delta(t) D\left(t, t^{\prime}\right) X\left(t^{\prime}\right) \\
& +\frac{i}{2} \int_{t_{i}}^{t_{f}} d t \int_{t_{i}}^{t_{f}} d t^{\prime} \Delta(t) N\left(t, t^{\prime}\right) \Delta\left(t^{\prime}\right),
\end{aligned}
$$

where we have introduced the average and difference coordinates defined, respectively, by

$$
X(t) \equiv \frac{1}{2}\left[x^{\prime}(t)+x(t)\right], \quad \Delta(t) \equiv x^{\prime}(t)-x(t) .
$$

The kernels $D\left(t, t^{\prime}\right)$ and $N\left(t, t^{\prime}\right)$ are called dissipation and noise kernels, respectively, and are defined by $D=\Sigma_{j} D_{j}$ and $N=\sum_{j} N_{j}$ where

$$
\begin{aligned}
D_{j}\left(t, t^{\prime}\right)= & -\frac{i}{2}\left\langle\left[\Xi_{j}(t), \Xi_{j}\left(t^{\prime}\right)\right]\right\rangle, \\
N_{j}\left(t, t^{\prime}\right)= & \frac{1}{2}\left\langle\left\{\Xi_{j}(t), \Xi_{j}\left(t^{\prime}\right)\right\}\right\rangle-\left\langle\Xi_{j}(t)\right\rangle \\
& \times\left\langle\Xi_{j}\left(t^{\prime}\right)\right\rangle,
\end{aligned}
$$

with $\Xi_{j}=g q_{j}^{2}$. It is now convenient to introduce the kernels $H_{j}\left(t, t^{\prime}\right)=-2 D_{j}\left(t, t^{\prime}\right) \theta\left(t-t^{\prime}\right)$ and we can write the influence action in the form

$$
S_{I F}\left[x, x^{\prime}\right]=\Delta \cdot H \cdot X+\frac{i}{2} \Delta \cdot N \cdot \Delta,
$$

where we have introduced the notation $A \cdot B \equiv \int d t A(t) B(t)$ and defined $H=\Sigma_{j} H_{j}$ which we may write formally as $H\left(t, t^{\prime}\right)=-2 D\left(t, t^{\prime}\right) \theta\left(t-t^{\prime}\right)$. This last equality is however a formal expression since being the product of two distributions, $H$ is not well defined and suitable regularization and renormalization are required. This term, in fact, has local divergent parts that may be reabsorbed into the parameters of the bare action, see [66] for details. Thus, from now on we will assume that $H$ is a well defined distribution in the previous sense. 


\section{B. The reduced Wigner function}

Our main purpose in this subsection is to write the reduced Wigner function for the system in a suitable way. This is a phase space function which is defined from the reduced density matrix by the following integral transform

$$
f(X, p, t)=\frac{1}{2 \pi \hbar} \int_{-\infty}^{\infty} d \Delta e^{i p \Delta / \hbar} \rho_{r}(X-\Delta / 2, X+\Delta / 2, t) .
$$

The reduced density matrix (8) at time $t_{f}$ can be computed from the path integrals of Eq. (9). To carry out this computation we will follow closely Ref. [67] where a similar computation for a linear system coupled linearly to an environment was described. For this reason we will describe here only the main steps and will concentrate on those which are peculiar to the nonlinear system. This is performed in several steps in which a key role is played by the use of the coordinates $X$ and $\Delta$ instead of $x$ and $x^{\prime}$. The first step is to integrate the system action in Eq. (9) by parts using the new coordinates $X$ and $\Delta$,

$$
\begin{aligned}
S[x]-S\left[x^{\prime}\right]= & -\left.\dot{X} \Delta\right|_{t_{i}} ^{t_{f}}+\int_{t_{i}}^{t_{f}} d t \Delta(t)\left(\frac{d^{2}}{d t^{2}} X(t)+\left.\frac{\partial V}{\partial X}\right|_{\Delta=0}\right) \\
& +\ldots,
\end{aligned}
$$

where the ellipsis stands for the terms nonlinear in $\Delta$ that come from the potential $V(x)$ which involve higher derivatives in $X$ (see below). Note that due to the fact that the potential gradient is evaluated at $\Delta=0$ this term can also be written as $V^{\prime}(X)$ where $V(X)$ is functionally the same as $V(x)$.

The change of integration variables $\int_{x_{i}}^{x_{f}} \mathcal{D} x \int_{x_{i}^{\prime}}^{x_{f}^{\prime}} \mathcal{D} x^{\prime}$ $\rightarrow \int_{X_{i}}^{X_{f}} D X \int_{\Delta_{i}}^{\Delta_{f}} \mathcal{D} \Delta$ involves a Jacobian which is unity and thus the path integration of the propagator (9) can be written as

$$
\int_{X_{i}}^{X_{f}} \mathcal{D} X \int_{\Delta_{i}}^{\Delta_{f}} \mathcal{D} \Delta e^{(i / \hbar) \Delta \cdot L[X]} F[\Delta, X]
$$

where $L[X]$ is a functional of $X$ and a function of $t$ defined by

$$
L[X ; t) \equiv\left(\frac{d^{2}}{d t^{2}} X(t)+\left.\frac{\partial V}{\partial X}\right|_{\Delta=0}\right)+\int_{t_{i}}^{t} d t^{\prime} H\left(t, t^{\prime}\right) X\left(t^{\prime}\right),
$$

and the functional $F[\Delta, X]$ incorporates in the exponent all the terms that are not linear in $\Delta$ which come from the influence action and from the nonlinear potential of the system action, when expressed in the variables $X(t)$ and $\Delta(t)$. More precisely,

$$
F[\Delta, X]=\exp _{\hbar} \frac{i}{\hbar}\left(\frac{i}{2} \Delta \cdot N \cdot \Delta+\int_{t_{i}}^{t} d t^{\prime} V^{(n l)}[\Delta, X]\right),
$$

where

$$
V^{(n l)}[\Delta, X]=\left.2 \sum_{n \geqslant 1} \frac{1}{(2 n+1) !} \frac{\partial^{(2 n+1)} V}{\partial X^{(2 n+1)}}\right|_{X, \Delta=0}(-\Delta / 2)^{2 n+1}
$$

In particular, for the cubic potential $V^{(n l)}(x)=-(\lambda / 6) x^{3}$ we have $V^{(n l)}[\Delta, X]=-(\lambda / 24) \Delta^{3}$.

Let us now introduce the functional Fourier transform

$$
P[\xi]=K \int \mathcal{D} \Delta e^{i \Delta \cdot \xi / \hbar} e^{-\Delta \cdot N \cdot \Delta / 2 \hbar},
$$

where $K=1 / \operatorname{det}(2 \pi \hbar I)$, the interpretation of this functional will be discussed below. With expression (22) we may write the reduced Wigner function as

$$
\begin{aligned}
f\left(X_{f}, p_{f}, t_{f}\right)= & \frac{1}{2 \pi \hbar} \int d X_{i} d \Delta_{i} \int \mathcal{D} \xi P[\xi] \\
& \times \int_{-\infty}^{\infty} d \Delta_{f} e^{i p_{f} \Delta_{f} / \hbar} \int_{X_{i}}^{X_{f}} \mathcal{D} X e^{-i \Delta_{f} \dot{X}\left(t_{f}\right) / \hbar} \\
& \times \int_{\Delta_{i}}^{\Delta_{f}} \mathcal{D} \Delta e^{i \Delta \cdot(L[X]-\xi) / \hbar} e^{i \int V^{(n l) / \hbar}} e^{i \Delta_{i} \dot{X}\left(t_{i}\right) / \hbar} \\
& \times \rho_{r}\left(X_{i}-\Delta_{i} / 2, X_{i}+\Delta_{i} / 2, t_{i}\right)
\end{aligned}
$$

and using that $\Delta=\exp (i \Delta \cdot \xi / \hbar)(i \hbar \delta / \delta \xi) \exp (-i \Delta \cdot \xi / \hbar)$, it may be rewritten as

$$
\begin{aligned}
f\left(X_{f}, p_{f}, t_{f}\right)= & \frac{1}{2 \pi \hbar} \int d X_{i} d \Delta_{i} \int_{X_{i}}^{X_{f}} \mathcal{D} X \int \mathcal{D} \xi P_{Q}\left[\xi, X ; t_{f}\right) \\
& \times \int_{-\infty}^{\infty} d \Delta_{f} e^{i\left[p_{f}-\dot{X}\left(t_{f}\right)\right] \Delta_{f} / \hbar} \\
& \times \int_{\Delta_{i}}^{\Delta_{f}} \mathcal{D} \Delta e^{i \Delta \cdot(L[X]-\xi) / \hbar} e^{i \Delta_{i} \dot{X}\left(t_{i}\right) / \hbar} \\
& \times \rho_{r}\left(X_{i}-\Delta_{i} / 2, X_{i}+\Delta_{i} / 2, t_{i}\right),
\end{aligned}
$$

where

$$
P_{Q}[\xi, X ; t)=\left\{\exp \left[\frac{i}{\hbar} \int_{t_{i}}^{t} d t^{\prime} V^{(n l)}\left[-i \hbar \frac{\delta}{\delta \xi}, X\right]\right]\right\} P[\xi] .
$$

A convenient way to perform the path integration for $X(t)$ is to introduce the following functional change:

$$
X(t) \rightarrow\left\{X_{i}=X\left(t_{i}\right), p_{i}=\dot{X}\left(t_{i}\right), \widetilde{\xi}(t)=L[X ; t)\right\} .
$$

With this transformation the function $X(t)$ becomes substituted by the initial conditions $\left(X_{i}, p_{i}\right)$ and the function $\widetilde{\xi}(t)$ in the path integration. This functional change is invertible, in the sense that $\left\{X_{i}, p_{i}, \widetilde{\xi}(t)\right\} \rightarrow X(t)$, since the solution $X(t)$ of the integro-differential equation involved in (24) is unique given initial conditions $\left(X_{i}, p_{i}\right)$. A subtler point concerns the Jacobian of the transformation (24). Even though this transformation is nonlinear, one can show that the Jacobian is 
constant. This can be seen by discretizing the time $t_{k}=\epsilon k$ $+t_{i}\left(k=1,2, \ldots, n\right.$, and $t_{i}$ is the initial time). Then the corresponding values $\left(X_{i}, X_{1}, \ldots, X_{n}\right)$, where $X_{k}=X\left(t_{k}\right)$, map into $\left(X_{i}, p_{i}, \widetilde{\xi}_{2}, \ldots, \widetilde{\xi}_{n}\right)$ in such a way that the Jacobian matrix has zero elements above the diagonal. For instance, the second derivative terms become $\left[\left(X_{k}-X_{k-1}\right)-\left(X_{k-1}\right.\right.$ $\left.\left.-X_{k-2}\right)\right] / \epsilon^{2}+V^{\prime}\left(X_{k-1}\right)-\Sigma_{k^{\prime}<k} H_{k k^{\prime}} X_{k^{\prime}}=\widetilde{\xi}_{k}$. The Jacobian is thus the product of the diagonal elements, which are constant (independent of any $X_{k}$ ). Then one may write $\int \mathcal{D} X \ldots=\bar{K} \int d X_{i} d p_{i} \int \mathcal{D} \tilde{\xi} \ldots$ and introduce convenient delta functions such as $\delta\left(X\left(t_{f}\right)-X_{f}\right)$ to ensure that the correct final points appearing in (18), i.e., $\int^{X_{f}} \mathcal{D} X$ are recovered from the functional integral $\int \mathcal{D} \widetilde{\xi}$ with free ends. One should also be careful about the dependence on the initial conditions $\left(X_{i}, p_{i}\right)$ in the general case.

Now we first perform the integral $\mathcal{D} \Delta$ which simply leads to a term proportional to $\delta(\widetilde{\xi}-\xi)$ and the integral $\mathcal{D} \widetilde{\xi}$ is then trivial. On the other hand the integral $d \Delta_{i}$ brings back the reduced Wigner function at the initial time according to Eq. (16). Finally, we get the following suggestive form for the reduced Wigner function at the final time

$$
\begin{aligned}
f\left(X_{f}, p_{f}, t_{f}\right)= & \bar{K} \int d X_{i} d p_{i} \int \mathcal{D} \xi P_{Q}\left[\xi ; t_{f}\right) \delta\left(p_{f}-\dot{X}\left(t_{f}\right)\right) \\
& \times \delta\left(X_{f}-X\left(t_{f}\right)\right) f\left(X_{i}, p_{i}, t_{i}\right),
\end{aligned}
$$

where $X(t)$ is a solution of the integro-differential equation

$$
L[X ; t)=\xi(t),
$$

with initial conditions $\left(X_{i}, p_{i}\right)$, i.e. $X=X\left[\xi ; X_{i}, p_{i}\right)$, and

$$
P_{Q}\left[\xi ; X_{i}, p_{i}, t\right)=P_{Q}\left[\xi, X\left[\xi ; X_{i}, p_{i}\right) ; t\right) .
$$

The constant $\bar{K}$ from the Jacobian can be determined from the condition that $\operatorname{Tr} \rho_{r}\left(t_{f}\right)=1$, i.e. that $\int_{-\infty}^{\infty} d X_{f} \rho_{r}\left(X_{f}, X_{f}, t_{f}\right)=1$, which is equivalent to $\int_{-\infty}^{\infty} d X_{f} d p_{f} f\left(X_{f}, p_{f}, t_{f}\right)=1$. Inserting expression (25) for $f\left(X_{f}, p_{f}, t_{f}\right)$ we get

$$
\bar{K} \int d X_{i} d p_{i} \int \mathcal{D} P_{Q}\left[\xi ; t_{f}\right) f\left(X_{i}, p_{i}, t_{i}\right)=1 .
$$

When $P_{Q}[\xi, X ; t)$ does not depend on $X$, one can use the fact that $\int_{-\infty}^{\infty} d X_{i} \int_{-\infty}^{\infty} d p_{i} f\left(X_{i}, p_{i}, t_{i}\right)=1$, which is a consequence of $\operatorname{Tr} \rho_{r}\left(t_{i}\right)=1$ to obtain,

$$
\bar{K} \int \mathcal{D} \xi P_{Q}[\xi ; t)=\bar{K} \int \mathcal{D} \xi P[\xi]=1,
$$

which determines $\bar{K}$.

Several remarks are in order here. The functional $P_{Q}\left[\xi, X\left[\xi ; X_{i}, p_{i}\right) ; t\right)$ is always real, but in general it will not be positive definite and, thus, will not really correspond to the probability density functional for a classical stochastic process. This is the meaning that must be associated to the stochastic process in the Langevin-like equation (26). This situation is, in fact, analogous to that for the Wigner function but applied here to distribution functionals. Note that this is in contrast to the linear case studied in Ref. [67], where the source of the Langevin equation really corresponded to a stochastic process (with a positive probability density functional).

We emphasize again that if we have a cubic potential for the system, $V(x)=-(\lambda / 6) x^{3}$, and keep up to quadratic order in $g$, we have explicitly

$$
F[\Delta, X]=e^{-(1 / 2 \hbar) \Delta \cdot N \cdot \Delta} e^{-(i / \hbar) \int d t(\lambda / 24) \Delta^{3}},
$$

where the noise kernel $N$ and the kernel $H$, which appeared in $L$ above, are both quadratic in $g$; note that there is no dependence on $X$ in this case, and thus $P_{Q}[\xi ; t)$ defined in Eq. (27) does not depend on the initial conditions $\left(X_{i}, p_{i}\right)$. $\mathrm{Hu}, \mathrm{Paz}$ and Zhang [68] obtained the master equation for the particular case in which the nonlinear potential of the system is also treated perturbatively in $\lambda$, which was considered to be of the same order as $g$. Here, however, our result is exact in $\lambda$, and to leading order in $\hbar$. This fact will turn out to be important since in Sec. IV it will be crucial to consider solutions of the classical equations of motion which are nonperturbative in $\lambda$ thus reflecting their strong nonharmonicity.

\section{The master equation}

The expression (25) of the reduced Wigner function and the Langevin-like equation (26) can be used to derive the master equation for $f$ as a formal Fokker-Planck equation. The derivation is usually handled using Novikov's formula when the stochastic process is Gaussian $[82,73,67]$. Here, however, this is not the case for $P_{Q}$ and we have to work from the beginning.

To obtain the equation of motion for the Wigner function, we derive both terms of Eq. (25) with respect to time. Observe that $P_{Q}$ depends explicitly on time, therefore

$$
\frac{\partial}{\partial t_{f}} f\left(X_{f}, p_{f}, t_{f}\right)=A+B,
$$

where

$$
\begin{aligned}
A= & \bar{K} \int d X_{i} d p_{i} \int \mathcal{D} \xi P_{Q}\left[\xi ; t_{f}^{-}\right)\left(\frac { \partial } { \partial t _ { f } } \left[\delta\left(p_{f}-\dot{X}\left(t_{f}\right)\right)\right.\right. \\
& \left.\left.\times \delta\left(X_{f}-X\left(t_{f}\right)\right)\right]\right) f\left(X_{i}, p_{i}, t_{i}\right)
\end{aligned}
$$

(we write $t_{f}^{-}$in $P_{Q}$ to emphasize that the dependence on $t$ is taken care of explicitly by the $B$ term) and

$$
\begin{aligned}
B= & \bar{K} \int d X_{i} d p_{i} \int \mathcal{D} \xi\left(\frac{\partial}{\partial t_{f}} P_{Q}\left[\xi ; t_{f}\right)\right) \delta\left(p_{f}-\dot{X}\left(t_{f}\right)\right) \\
& \times \delta\left(X_{f}-X\left(t_{f}\right)\right) f\left(X_{i}, p_{i}, t_{i}\right) .
\end{aligned}
$$

Let us analyze the $B$ term first. Since

$$
\frac{\partial}{\partial t_{f}} P_{Q}\left[\xi ; t_{f}\right)=\frac{i}{\hbar} V^{(n l)}\left[-i \hbar \frac{\delta}{\delta \xi\left(t_{f}\right)}, X_{f}\right] P_{Q}\left[\xi ; t_{f}^{-}\right),
$$


integrating by parts we find,

$$
\begin{aligned}
B= & \bar{K} \int d X_{i} d p_{i} \int \mathcal{D} \xi P_{Q}\left[\xi ; t_{f}\right)\left[\frac{i}{\hbar} V^{(n l)}\left[i \hbar \frac{\delta}{\delta \xi\left(t_{f}\right)}, X_{f}\right]\right. \\
& \times\left[\delta\left(p_{f}-\dot{X}\left(t_{f}\right)\right) \delta\left(X_{f}-X\left(t_{f}\right)\right)\right] f\left(X_{i}, p_{i}, t_{i}\right)
\end{aligned}
$$

We are only interested in derivatives taken at $t_{f}$, when

$$
\frac{\delta X\left(t_{f}\right)}{\delta \xi\left(t_{f}\right)}=0, \quad \frac{\delta \dot{X}\left(t_{f}\right)}{\delta \xi\left(t_{f}\right)}=1
$$

without further dependence on $\xi\left(t_{f}\right)$. This can be seen from the fact that $\delta X(t) / \delta \xi\left(t^{\prime}\right)$ satisfies $[(\bar{L}+H) \cdot \delta X / \delta \xi]\left(t, t^{\prime}\right)$ $=\delta\left(t-t^{\prime}\right)$ with the $X(t)$ which appears in $\bar{L}$ fixed, $\bar{L}$ is the integro-differential operator $\bar{L}\left(t, t^{\prime}\right) \equiv\left(d^{2} / d t^{2}+\partial^{2} V /\right.$ $\left.\left.\partial X^{2}\right|_{X^{(0)}}\right) \delta\left(t-t^{\prime}\right)$; see Eq. (26). The solution is $\delta X(t) / \delta \xi\left(t^{\prime}\right)=G_{r e t}\left(t, t^{\prime}\right)$, which is the retarded propagator corresponding to the linear operator $(\bar{L}+H)$ with $X(t)$ fixed. So the final result is

$$
B=\frac{i}{\hbar} V^{(n l)}\left[-i \hbar \frac{\delta}{\delta p_{f}}, X_{f}\right] f\left(X_{f}, p_{f}, t_{f}\right) .
$$

Concerning $A$, we find

$$
\begin{aligned}
A= & -\bar{K} \int d X_{i} d p_{i} \int \mathcal{D} \xi P_{Q}\left[\xi ; t_{f}^{-}\right)\left[\left(\frac{d X\left(t_{f}\right)}{d t_{f}} \frac{\partial}{\partial X_{f}}\right.\right. \\
& \left.\left.+\frac{d \dot{X}\left(t_{f}\right)}{d t_{f}} \frac{\partial}{\partial p_{f}}\right)\left[\delta\left(p_{f}-\dot{X}\left(t_{f}\right)\right) \delta\left(X_{f}-X\left(t_{f}\right)\right)\right]\right] f_{i},
\end{aligned}
$$

and reading the derivatives from Eq. (26) we can write $A$ $=A_{1}+A_{2}+A_{3}$. The first term is simply

$$
A_{1}=\left\{H_{s}, f\right\}
$$

where $\left\{H_{s}, f\right\}=-p(\partial f / \partial X)+V^{\prime}(\partial f / \partial p)$ is the Poisson bracket with $H_{s}=p^{2} / 2+V(X)$ the system Hamiltonian. The second term is

$$
\begin{aligned}
A_{2}= & -\frac{\partial}{\partial p_{f}} \bar{K} \int d X_{i} d p_{i} \int \mathcal{D} \xi P_{Q}\left[\xi ; t_{f}^{-}\right) \\
& \times\left[\int_{t_{i}}^{t_{f}} d t^{\prime} H\left(t_{f}, t^{\prime}\right) X\left(t^{\prime}\right)\right]\left[\delta\left(p_{f}-\dot{X}\left(t_{f}\right)\right)\right. \\
& \left.\times \delta\left(X_{f}-X\left(t_{f}\right)\right)\right] f_{i},
\end{aligned}
$$

to lowest order in $\hbar$, we are entitled to replace $X\left(t^{\prime}\right)$ inside the non-local term by a solution of the classical equations of motion with the given Cauchy data $X_{f}$ and $p_{f}$. We shall call this procedure of substitution of the classical trajectories into the terms which are already of order $\hbar$ "reduction of order." We may then extract the non-local term from the integral to get the simpler form

$$
A_{2}=\frac{\partial}{\partial p}[\Gamma(X, p, t) f],
$$

where $\Gamma(X, p, t)=-\int_{t_{i}}^{t} d t^{\prime} H\left(t, t^{\prime}\right) X\left(t^{\prime}\right)$. Finally, the third term is

$$
\begin{aligned}
A_{3}= & -\frac{\partial}{\partial p_{f}} \bar{K} \int d X_{i} d p_{i} \int \mathcal{D} \xi P_{Q}\left[\xi: t_{f}^{-}\right) \xi\left(t_{f}\right) \\
& \times \delta\left(p_{f}-\dot{X}\left(t_{f}\right)\right) \delta\left(X_{f}-X\left(t_{f}\right)\right) f_{i} .
\end{aligned}
$$

To compute this term, we note from Eqs. (23) and (27) that

$$
\begin{aligned}
\xi\left(t_{f}\right) P_{Q}\left[\xi ; t_{f}^{-}\right)= & \left\{\exp \left[\frac{i}{\hbar} \int t_{f}^{-} d t^{\prime} V^{(n l)}\left[-i \hbar \frac{\delta}{\delta \xi}, X\right]\right]\right\} \\
& \times \xi\left(t_{f}\right) P[\xi]
\end{aligned}
$$

and since $P[\xi]$ is Gaussian, we may use the identity (Novikov's formula):

$$
\xi\left(t_{f}\right) P_{Q}\left[\xi: t_{f}^{-}\right)=-\hbar \int_{t_{i}}^{t} d t^{\prime} N\left(t, t^{\prime}\right) \frac{\delta}{\delta \xi\left(t^{\prime}\right)} P_{Q}\left[\xi ; t_{f}^{-}\right)
$$

Integrating Eq. (42) by parts and after further simplification, where we explicitly assume that, as in the case of the cubic potential, $P_{Q}$ is independent of $X$ (in this way we can commute the exponential of $V^{(n l)}$ in $P_{Q}$ with the functional derivative with respect to $\xi$ ), we obtain

$$
A_{3}=\frac{\partial}{\partial p_{f}}\{N, f\}
$$

where $N \equiv \hbar \int d t^{\prime} N\left(t, t^{\prime}\right) X\left(t^{\prime}\right)$, and we have applied once again a reduction of order prescription. The details of this calculation are given in Appendix A.

To summarize, and using the explicit form of $V^{(n l)}$ for the cubic potential, we obtain the following dynamical equation for the reduced Wigner function (master equation):

$$
\frac{\partial f}{\partial t}=\left\{H_{s}, f\right\}+\frac{\partial}{\partial p}[\Gamma(X, p, t) f]+\frac{\partial}{\partial p_{f}}\{N, f\}-\hbar^{2} \frac{\lambda}{24} \frac{\partial^{3} f}{\partial p^{3}}
$$

If the system were isolated, the master Eq. (46) would reduce to

$$
\frac{\partial W}{\partial t}=\left\{H_{s}, W\right\}-\hbar^{2} \frac{\lambda}{24} \frac{\partial^{3} W}{\partial p^{3}},
$$

where $W$ is the Wigner function of the closed system, $H_{s}$ its Hamiltonian and the curly brackets are the Poisson brackets. This equation is exactly equivalent to von Neumann's equation for the density matrix of a one-dimensional quantum mechanical system with a cubic potential $V(x)=-(\lambda / 6) x^{3}$. Note that the term with the third derivative with respect to the momentum is responsible for tunneling when properly combined with the otherwise classical dynamics generated 
by the term corresponding to the Poisson bracket, i.e., if this term were not present, the evolution of the Wigner function would be entirely equivalent to that of a classical ensemble in phase space.

There is a theorem by Pawula [69] which states that a diffusion-like equation such as Eq. (47) should have up to second order derivatives at most, or else an infinite KramersMoyal expansion, for non-negative solutions $W(x, p, t)$ to exist. The equation for the Wigner function circumvents the implications of the theorem since it need not be everywherepositive. Even if we have an everywhere-positive Gaussian Wigner function at the initial time, the evolution generated by an equation such as Eq. (47) will not keep it everywherepositive. This can be connected with the fact that the Wigner function could be interpreted as the distribution function associated to an ensemble of solutions satisfying the Langevinlike equation (26) with a generalized stochastic source $\xi(t)$ with a non-positive probability density functional $P_{Q}[\xi]$. Thus, here we see the essential role played by the nonpositivity of the Wigner function in a genuinely quantum aspect such as tunneling. In other aspects such as in quantum coherence this role is well known [70].

It is important to stress the following points. First, we are not assuming that the stochastic trajectories described by the Langevin equation are real trajectories; although they may be if there is decoherence [71]. Second, although we use the initial Wigner function to weight the initial conditions, we do not assume it is a probability distribution function. Our interest in the Wigner function is that we shall use the fact that it acquires a substantial nonzero average beyond the basin of attraction of the false vacuum as a signal that tunneling has occurred. This application is valid even if the Wigner function itself cannot be understood as a probability distribution function, because the distributions computed from the Wigner functions, such as $\int_{-\infty}^{\infty} d X f(X, p)$ or $\int_{-\infty}^{\infty} d p f(X, p)$ are true probability distributions, and $\int_{-\infty}^{\infty} d X(p / M) f(X, p)$ is a true probability flux [72].

\section{Dynamics of the distribution function $f$}

To compute the tunneling probability from Eq. (47) is possible using, for instance, a WKB approximation scheme [20,21]. Alternatively one may use the instanton method [9] which gives a simple answer in this case. Our main interest in this paper is not this contribution to tunneling but rather to compute the effect due to the backreaction of the environment. To simplify our derivation we shall assume that the time scales are different, that the dominant term is the backreaction effect and, thus, we will neglect the third derivative term in the master equation (46). More precisely, we will use the evolution equation for the distribution function $f$

$$
\frac{\partial f}{\partial t}=\left\{H_{s}, f\right\}+\frac{\partial}{\partial p}[\Gamma(X, p, t) f]+\frac{\partial}{\partial p_{f}}\{N, f\} .
$$

This equation describes an ensemble of points evolving according to the dynamics

$$
\dot{X}=p, \quad \dot{p}=-V^{\prime}(X)+\int_{-\infty}^{t} d t^{\prime} H\left(t-t^{\prime}\right) X\left(t^{\prime}\right)+\xi,
$$

where $H$ is the dissipation kernel introduced in Eq. (15), and $\xi$ is a Gaussian noise described by the noise kernel $N$ introduced in Eq. (11), as

$$
\left\langle\xi(t) \xi\left(t^{\prime}\right)\right\rangle=\hbar N\left(t-t^{\prime}\right) .
$$

Although we set up the initial conditions at $t_{i}=0$, we extend the lower integration limit in Eq. (49) to $-\infty$ for computational purposes. The approximation is, nevertheless, justified since the characteristic frequencies of the environment, $\omega_{j} \gtrless 1$ (recall that the system particle has unit mass), are much larger than the typical decay rate of the initial false vacuum state. In other words, the characteristic time scale for the environment dynamics $t \lesssim 1$ and hence, the typical "correlation time" for the dissipation and noise kernels, is much smaller than the typical decay time. The contribution to the integration interval $(-\infty, 0)$ is, therefore, relatively small.

According to Eqs. (13) and (14) these kernels admit the following representation,

$$
\begin{aligned}
& N\left(t-t^{\prime}\right)=\frac{1}{2}\left\langle\left\{\Xi(t), \Xi\left(t^{\prime}\right)\right\}\right\rangle-\langle\Xi(t)\rangle\left\langle\Xi\left(t^{\prime}\right)\right\rangle, \\
& H\left(t-t^{\prime}\right)=\frac{i}{\hbar}\left\langle\left[\Xi(t), \Xi\left(t^{\prime}\right)\right]\right\rangle \theta\left(t-t^{\prime}\right),
\end{aligned}
$$

where $\Xi \equiv \Sigma_{j} \Xi_{j}$ and where we must keep in mind the need for regularization of the kernel $H$ as defined above. Let us take the Fourier transforms,

$$
\begin{gathered}
\left\langle\left[\Xi(t), \Xi\left(t^{\prime}\right)\right]\right\rangle=\int \frac{d \omega}{2 \pi} e^{-i \omega\left(t-t^{\prime}\right)} \hbar \omega \gamma(\omega), \\
N\left(t-t^{\prime}\right)=\int \frac{d \omega}{2 \pi} e^{-i \omega\left(t-t^{\prime}\right)} \nu(\omega) .
\end{gathered}
$$

For an environment initially in thermal equilibrium at temperature $T=\beta^{-1}$ the functions $\gamma$ and $\nu$ will be related through the fluctuation-dissipation theorem

$$
\nu(\omega)=\left[\frac{1}{2}+f_{0}(\omega)\right] \hbar|\omega| \gamma(\omega), \quad f_{0}(\omega)=\left(e^{\beta \hbar|\omega|}-1\right)^{-1},
$$

which is a consequence of the Kubo-Martin-Schwinger (KMS) formula. Here we shall consider the zero temperature case only.

In order to compute the memory terms in Eq. (49), it is convenient to parametrize the trajectories by their initial conditions at time $t_{i}=0$. These trajectories may be written in terms of the action-angle variables $J$ and $\theta$ associated to the classical potential, which we assume has a potential well bounded by a finite potential barrier. In other words, we are using $J$ and $\theta$ as Lagrangian coordinates, identifying a given trajectory, while $X$ and $p$ are like Eulerian coordinates, identifying where the trajectory is at a given time. The action 
variable is defined by $J=(1 / 2 \pi) \oint p d X$; since $p$ can be written in terms of $\dot{X}$ and the system Hamiltonian $H_{s}$, substitution into the equation defining $J$ and inversion implies that $H_{s}=H_{s}(J)$, and $d H_{s} / d J=\Omega(J)$ is the frequency of the motion. The angle variable $\theta$ changes from 0 to $2 \pi$ and satisfies the equation of motion $\dot{\theta}=\Omega$.

Since the kernel $H$ is already of order $\hbar$, in the memory term of Eq. (49) we must replace the trajectory $X\left(t^{\prime}\right)$ by a solution of the classical equation of motion, in which case the transformation to variables $(J, \theta)$ is canonical. For fixed $J$, the classical trajectory is periodic, $X\left(t^{\prime}\right)=X\left(t^{\prime}+2 \pi / \Omega\right)$; note that the motion is periodic inside the potential well but at higher energies, near the top of the potential barrier (the separatrix) when $J \rightarrow J_{s}$, the motion ceases to be periodic and $\Omega \rightarrow 0$. Thus we may write

$$
\begin{aligned}
& X\left(t^{\prime}\right)=\sum_{n} e^{i n\left[\theta+\Omega(J) t^{\prime}\right]} X_{n}(J) \\
& p\left(t^{\prime}\right)=i \Omega(J) \sum_{n} e^{i n\left[\theta+\Omega(J) t^{\prime}\right]} n X_{n}(J)
\end{aligned}
$$

where $X_{-n}=X_{n}^{*}$, since $X$ is real. We then write the memory dependent term as:

$$
\begin{aligned}
\Gamma & =-\int_{-\infty}^{t} d t^{\prime} H\left(t-t^{\prime}\right) X\left(t^{\prime}\right) \\
& =-\sum_{n} X_{n}(J) e^{i n[\theta+\Omega(J) t]} \int \frac{d \omega}{2 \pi} \frac{\omega \gamma(\omega)}{\omega+n \Omega(J)-i \varepsilon},
\end{aligned}
$$

where we have used Eqs. (52), (53) and (56), and that $\int_{0}^{\infty} d u \exp (i s u)=i /(s+i \varepsilon)$. Therefore we have

$$
\begin{gathered}
\Gamma(X, p, t)=-\sum_{n} X_{n}(J) e^{i n[\theta+\Omega(J) t]} \gamma_{n}(J), \\
\gamma_{n}(J)=\int \frac{d \omega}{2 \pi} \frac{\omega \gamma(\omega)}{\omega+n \Omega(J)-i \varepsilon} .
\end{gathered}
$$

Observe that although the Langevin equation is now local in time, it is not necessarily Ohmic. A similar manipulation of the last term in the master equation (48) gives (see Appendix A)

$$
\begin{gathered}
N(J, \theta)=\sum_{n} X_{n}(J) e^{i n[\theta+\Omega(J) t]} N_{n}(J), \\
N_{n}(J)=\int \frac{d \omega}{2 \pi} \frac{[-i \nu(\omega)]}{\omega+n \Omega(J)-i \varepsilon} .
\end{gathered}
$$

\section{E. Weak dissipation limit: Averaging over angles}

So far, we have kept $f$ arbitrary. To study tunneling, however, we may impose the additional condition that $f=f(J)$, and obtain a simpler equation by averaging the Fokker-
Planck equation over the angle variable $\theta$. This approximation has been discussed by Kramers [7,69] as prevailing in the weak dissipation limit. Recall that then $\left\{H_{s}, f\right\}=0$, and that, for any phase space function $\Psi(\theta, J)$,

$$
\begin{aligned}
\oint d \theta\{X, \Psi\} & =\oint d X\left\{\left.\frac{\partial \Psi}{\partial J}\right|_{\theta}-\left.\frac{\partial X}{\partial J}\right|_{\theta} \frac{\Psi}{\dot{X}}\right\} \\
& =\frac{d}{d J} \oint d X \Psi=\frac{d}{d J}\left[\frac{1}{\Omega} \oint d \theta p \Psi\right],
\end{aligned}
$$

where we have used that for the classical trajectory $d \theta$ $=\dot{\theta} d t=\Omega d t$, and that $d X=\left.\partial_{\theta} X\right|_{J} d \theta+\left.\partial_{J} X\right|_{\theta} d J$ from where by imposing $d X=0$ we can deduce $\left.\partial_{J} \theta\right|_{X}$.

Finally the Fokker-Planck equation (48) becomes

$$
\frac{\partial f}{\partial t}=\frac{d}{d J}\left\{\mathcal{N} \frac{d f}{\Omega d J}+\mathcal{D} f\right\}
$$

where we introduced $\mathcal{D}$ and $\mathcal{N}$ as follows:

$$
\begin{aligned}
& \mathcal{D} \equiv \frac{1}{\Omega} \oint d \theta p \Gamma=i \sum_{n}\left|X_{n}(J)\right|^{2} n \gamma_{n}(J), \\
& \mathcal{N} \equiv \oint d \theta p \frac{\partial N}{\partial \theta}=\Omega \sum_{n}\left|X_{n}(J)\right|^{2} n^{2} N_{n}(J) .
\end{aligned}
$$

Now observe that from Eq. (58) and using that $1 /(s+i \varepsilon)$ $=\mathrm{PV}(1 / s)+i \pi \delta(s)$ we can write

$$
\gamma_{n}(J)=\mathrm{PV} \int \frac{d \omega}{2 \pi} \frac{\omega \gamma(\omega)}{\omega+n \Omega(J)}-\frac{i}{2} n \Omega(J) \gamma[n \Omega(J)],
$$

but since $\quad 1 /[\omega-n \Omega(J)]-1 /[\omega+n \Omega(J)]=2 n \Omega /\left[\omega^{2}\right.$ $\left.-n^{2} \Omega^{2}(J)\right]$ the first term of $\gamma_{n}$ above integrates to zero, so that only the imaginary term contributes, and we finally have

$$
\mathcal{D}=\frac{\Omega}{2} \sum_{n}\left|X_{n}(J)\right|^{2} n^{2} \gamma[n \Omega] .
$$

A similar computation using expression (59) for $N_{n}$ leads to the final expression for $\mathcal{N}$ :

$$
\mathcal{N}=\frac{\Omega}{2} \sum_{n}\left|X_{n}(J)\right|^{2} n^{2} \nu[n \Omega] .
$$

\section{F. A rough estimate of the decay rate}

Equations (48) and (60) are the basic equations for the rest of our analysis. The rest of the paper is devoted to the explicit computation of the $\mathcal{D}$ and $\mathcal{N}$ functions in a field theoretical problem, and to solving the dynamical equations therefrom. However, we may already at this point make an educated guess about the relationship between the decay rate predicted by these equations, and the usual quantum estimates.

The point is that, since these are after all equations similar to those discussed by Kramers [7], we may obtain a rough estimate of the transition amplitude by just plugging in 
Kramers' result for the flux. This is made from Eq. (60) which we may write as a continuity equation $\partial_{t} f+\partial_{J} K=0$ where the probability flux $K$ may be directly identified from the equation. Then one looks for stationary solutions with positive flux $K_{0}$, which must satisfy $(\mathcal{N} / \Omega) \partial_{J} f+\mathcal{D} f=-K_{0}$. From this equation one may estimate [imposing that the particle is in the potential well $\int^{J_{s}} f(J) d J \leqslant 1$, where $J_{s}$ is $J$ at the separatrix, i.e. the top of the potential barrier] using that $d E=\Omega d J$ the following upper bound for $K_{0}$ :

$$
K_{0} \sim e^{-B\left(E_{s}\right)}, \quad B=\int d E(\mathcal{D} / \mathcal{N}) .
$$

At high temperature, we have $\nu=\gamma k T, \mathcal{N}=k T \mathcal{D}, B$ $=E / k T$, and

$$
K_{0} \sim \exp \left(-E_{s} / k T\right)
$$

where $E_{s}$ is the energy at the separatrix, as expected [69]. At low temperature, see Eq. (55), $\nu=\hbar|\omega| \gamma / 2$. Now, because the sums which define both $\mathcal{N}$ and $\mathcal{D}$ are dominated by frequencies of the order of the curvature of the potential around the unstable fixed point $\omega \sim \sqrt{\left|V_{s}^{\prime \prime}\right|}$ (this being the time scale for the exponential approach to the unstable fixed point), then $\mathcal{N} \sim \hbar \sqrt{\left|V_{s}^{\prime \prime}\right|} \mathcal{D} / 2$, and thus

$$
K_{0} \sim \exp \left(-2 E_{s} / \hbar \sqrt{\left|V_{s}^{\prime \prime}\right|}\right) .
$$

We may compare this estimate with the usual WKB tunneling amplitude given by

$$
K_{0}(\text { tunnel }) \sim \exp \left(-\hbar^{-1} \int d x \sqrt{2 V(x)}\right) .
$$

If the integral is dominated by the peak at the unstable fixed point $X_{s}$, then $V \sim E_{s}-V_{s}^{\prime \prime}\left(X-X_{s}\right)^{2} / 2$ and the Euclidean trajectory may be parametrized as $X \sim X_{s}-\sqrt{2 E_{s} / V_{s}^{\prime \prime}} \cos \theta, 0$ $\leqslant \theta \leqslant \pi$, which gives $\int d x \sqrt{2 V(x)} \sim \pi E_{s} / \sqrt{V_{s}^{\prime \prime}}$.

We arrive at the remarkable conclusion that tunneling is comparable to the noise effect from the environment. Of course, our coarse estimates are not reliable for an accurate comparison and we must proceed to a more quantitative account.

\section{QUANTUM FIELDS AS OPEN SYSTEMS}

\section{A. The model and system-environment split}

We wish to study vacuum decay at zero and finite temperatures for a $3+1$ quantum massive scalar field $\Phi$ with action

$$
S[\Phi]=\int d^{4} x\left(-\frac{1}{2} \eta^{\mu \nu} \partial_{\mu} \Phi \partial_{\nu} \Phi-\frac{1}{2} M^{2} \Phi^{2}+\frac{1}{6} g \Phi^{3}\right),
$$

where the metric convention is $\eta^{\mu \nu}=\operatorname{diag}(-1,1,1,1)(\mu, \nu$ $=0,1,2,3)$. Although we keep $\hbar$ explicit, we set $c=1$. The mass $M$ has units of (length) $)^{-1}, \Phi$ has units of $M \sqrt{\hbar}$ and $g$ of $M / \sqrt{\hbar}$.
As discussed in the Introduction, we wish to focus on the behavior of the long wavelength modes of the field. Let us split the field $\Phi=\phi+\varphi$, where $\phi$ represents the long wavelength modes and $\varphi$ the short wavelength modes. To do that we introduce a length scale $\Lambda^{-1}$ which will be suitably fixed for our problem. To define $\phi(x)$ we take a window function $W\left(x^{\prime}-x\right)$ centered at the point $x$ with a width $\Lambda^{-1}$ and convolute the field $\Phi$ with it

$$
\phi(x)=\int d^{3} x^{\prime} W\left(x^{\prime}-x\right) \Phi\left(x^{\prime}\right),
$$

then, of course, $\varphi(x)=\Phi(x)-\phi(x)$. In this way the field at each point has two contributions, one corresponding roughly to scales larger than or of order $\Lambda^{-1}$ and the other to smaller scales. This has its correspondence in momentum space; we may define the Fourier transform of $\Phi$ by

$$
\Phi(x)=\int \frac{d^{3} k}{(2 \pi)^{3}} e^{i \vec{k} \cdot \vec{x}} \Phi_{\vec{k}}(t),
$$

the long wavelength modes now become $\phi_{\vec{k}}(t)$ $=\widetilde{W}(-\vec{k}) \Phi_{\vec{k}}(t)$, where $\widetilde{W}(\vec{k})$ is the Fourier transform of $W$, and for the short wavelength modes we have $\varphi_{\vec{k}}=\Phi_{\vec{k}}-\phi_{\vec{k}}$. It may be convenient to use a Gaussian window. In this way $\widetilde{W}$ is also Gaussian with width $\Lambda$, or sometimes it may be more convenient to take a step function for the Fourier transform of the window such as $\widetilde{W}(\vec{k})=\theta(\Lambda-k)$ where $k=|\vec{k}|$.

At this point $\phi$ is still a field, that is, it contains an infinite number of degrees of freedom. It is convenient to reduce the system to a single degree of freedom, such as we have discussed in Sec. III. One way to accomplish this is simply to enclose the field in a box of size $\Lambda^{-1}$; see [74]. The boundary conditions in this case would introduce an undesired discrete spectrum for the modes. From a physical point of view it is more satisfactory to proceed as follows. In a region of volume $\Lambda^{-3}$ we define the average field

$$
\bar{\phi}(t)=\Lambda^{3} \int_{\Lambda^{-3}} d^{3} x \phi(x) .
$$

Note that if we introduce the function $\rho(k / \Lambda)$ $=\Lambda^{3} \int_{\Lambda^{-3}} \exp (-i \vec{k} \cdot \vec{x})$, which satisfies $\rho(0)=1$, then in momentum space we have $\bar{\phi}(t)=(2 \pi)^{-3} \int d^{3} k \rho(k / \Lambda) \phi_{\vec{k}}$ so that $\bar{\phi}$ is made up of the modes of the field with $k \leqslant \Lambda$.

Now when the fields $\phi$ and $\varphi$ are substituted into Eq. (67) the action will be decomposed in three parts. One involves the field $\phi$ only, another the field $\varphi$ only, both with the same functional dependence as the original action and a third interacting part involves terms linear in $\varphi$ and the quadratic term $(1 / 2) g \phi \varphi^{2}$ which comes from the cubic term in Eq. (67). We will approximate the different terms as follows: $\int_{\Lambda^{-3}} d^{3} x \phi^{n}(x) \simeq \Lambda^{3} \bar{\phi}^{n}$ for any integer $n$ and $\int_{\Lambda^{-3}} d^{3} x \varphi(x)$ $\simeq 0$ since the short wavelength modes should average to zero. Thus, generally we have $\int_{\Lambda^{-3}} d^{3} x \phi^{n} f(\varphi)$ $\simeq \bar{\phi}^{n} \int_{\Lambda^{-3}} d^{3} x f(\varphi)$, where $f$ is an arbitrary polynomic function of $\varphi$. Since we want to focus in the single degree of 
freedom $\bar{\phi}(t)$, which is the averaged field associated to a certain region of volume $\Lambda^{-3}$, it will be convenient to restrict the volume integrals involving the field $\phi$ to that volume.

Up to this point the scale $\Lambda$ has been arbitrary. Now we need to fix it. Since the field $\phi$ is homogeneous in the region considered, the gradient terms should be negligible in front of the mass term, $(\vec{\nabla} \phi)^{2} \ll M^{2} \phi^{2}$ and this means that the momentum cutoff for the modes of the field $\phi$ should be $k$ $\ll M$, which implies that there is an upper bound for the scale $\Lambda: \Lambda \leqslant M$. We also want to be able to treat the field $\varphi$ perturbatively and that means that the field should be stable, in this case the cubic term $\varphi^{3}$ will be a two-loop order term and may be neglected to leading order in $\hbar$. We will see in a moment that the condition for $\varphi$ to be stable implies a lower bound for $\Lambda: \Lambda \geqslant M$. Thus for the system-environment split to be consistent we need $\Lambda \sim M$ and the volume is $M^{-3}$.

Finally the action (67) can be approximated as a systemenvironment interaction action $S[\Phi] \simeq S_{s}[\bar{\phi}]+S_{e}[\varphi]$ $+S_{\text {int }}[\bar{\phi}, \varphi]$ where

$$
\begin{aligned}
S_{s}[\bar{\phi}]= & M^{-3} \int d t\left(\frac{1}{2} \dot{\bar{\phi}}^{2}-\frac{1}{2} M^{2} \bar{\phi}^{2}+\frac{1}{6} g \bar{\phi}^{3}\right), \\
S_{e}[\varphi]= & \int d t \int_{k \geqslant M} \frac{d^{3} k}{(2 \pi)^{3}}\left[\frac{1}{2} \dot{\varphi}_{\vec{k}} \dot{\varphi}_{-\vec{k}}-\frac{1}{2}\left(k^{2}\right.\right. \\
& \left.\left.+M^{2}\right) \varphi_{\vec{k}} \varphi_{-\vec{k}}\right], \\
S_{\text {int }}[\bar{\phi}, \varphi]= & -\frac{1}{2} g \int d t \int_{M^{-3}} d^{3} x \bar{\phi} \varphi^{2},
\end{aligned}
$$

where we made a mode decomposition and integrated over the whole space volume in the environment action. Now the potential of the system

$$
V(\bar{\phi})=\frac{1}{2} M^{2} \bar{\phi}^{2}-\frac{1}{6} g \bar{\phi}^{3},
$$

has a stable fixed point at $\bar{\phi}=0$ and an unstable fixed point at $\bar{\phi}=\phi_{s} \equiv 2 g^{-1} M^{2}$. The former corresponds to zero energy, and the latter to $E=E_{s} \equiv M^{-1} \phi_{s}^{2} / 6$, in the volume $M^{-3}$. For intermediate energies, we may have bound and unbound states. They are separated by a potential barrier, which at zero energy extends from $\bar{\phi}=0$ to $\bar{\phi}=\phi_{\text {exit }} \equiv 3 \phi_{s} / 2$. At any given energy there will be three classical turning points $\phi_{L}$ $<0<\phi_{R}<\phi_{s}<\phi_{X} ; \quad$ as $E \rightarrow 0, \phi_{L}, \phi_{R} \rightarrow 0$ and $\phi_{X}$ $\rightarrow \phi_{\text {exit }}$, while when $E \rightarrow E_{s}, \quad \phi_{R}, \phi_{X} \rightarrow \phi_{s}$ and $\phi_{L} \rightarrow$ $-\phi_{s} / 2$. We are thus in the situation described in Sec. III, consequently according to the estimate at the end of the last subsection, Eq. (65), we expect here that the tunneling rate will be $K_{0} \sim \exp \left(-\alpha M^{2} / \hbar g^{2}\right)$ where $\alpha$ is a dimensionless parameter to be determined. Since the system is the averaged field $\bar{\phi}$, the tunneling rate is now per unit volume. When comparing with Sec. III note that the system coordinate $x$ and frequency $\Omega_{0}$ in the QBM model correspond here to the averaged field $\bar{\phi}$ and the field mass $M$, respectively; also here there is only one coupling parameter $\lambda=g$.

For the environment modes to be stable as required we should have from the previous actions $S_{e}$ and $S_{i n t}$ that $k^{2}$ $+M^{2}-g \bar{\phi} \geqslant 0$, and since the maximum value that $\bar{\phi}$ may take at the barrier is $\phi_{s}=2 g^{-1} M^{2}$, it is clear that $k \geqslant M$ and this gives the lower bound for $\Lambda$ mentioned above.

Let us now follow Sec. III and define the function $\Xi(t)$ which appears in the interaction action which is a key function to construct the dissipation and noise kernels, see $\Xi_{j}(t)$ in Eqs. (13) and (14), or $\Xi(t)$ in Eqs. (51) and (52). Thus we likewise define

$$
\Xi(t) \equiv M^{3} \int_{M^{-3}} d^{3} x \frac{1}{2} g \varphi^{2}(x),
$$

and the interaction action becomes $S_{i n t}=M^{-3} \int d t \bar{\phi}(t) \Xi(t)$; note that $\Xi(t)$ has units of $M^{3} \sqrt{\hbar}$. By analogy with Eq. (10) the influence functional becomes

$$
\begin{aligned}
e^{i S_{I F} / \hbar}= & \int D \varphi D \varphi^{\prime} \rho_{e i} \exp \frac{i}{\hbar}\left[S_{e}[\varphi]-S_{e}\left[\varphi^{\prime}\right]\right. \\
& \left.-M^{-3} \int d t\left[\bar{\phi}(t) \Xi(t)-\bar{\phi}^{\prime}(t) \Xi^{\prime}(t)\right]\right],
\end{aligned}
$$

where $\rho_{e i}$ is the environment density matrix at the initial time. As in the previous section, see Eq. (15), we keep only quadratic terms and we can write

$$
\begin{aligned}
S_{I F}= & \frac{1}{2} M^{-3}\left(\bar{\phi}-\bar{\phi}^{\prime}\right) \cdot H \cdot\left(\bar{\phi}+\bar{\phi}^{\prime}\right) \\
& +\frac{i}{2} M^{-3}\left(\bar{\phi}-\bar{\phi}^{\prime}\right) \cdot N \cdot\left(\bar{\phi}-\bar{\phi}^{\prime}\right) .
\end{aligned}
$$

Thus we are now in the situation described in general terms in Sec. III, and must complete the following steps: (a) identify the kernels $\gamma$ and $\nu$, (b) evaluate the Fourier transforms of $x$ and $p$ along a classical trajectory, (c) compute the functions $\mathcal{D}$ and $\mathcal{N}$, (d) solve the Fokker-Planck equation for $f$, or at least justify the approximations already discussed, and (e) evaluate the decay rate. We shall carry tasks (a), (b) and (c) in this section, and leave (d) and (e) for the next.

We should emphasize that as remarked in the previous section, the kernel $H$ needs to be regularized and that this involves a mass renormalization. We assume from now on that the mass $M$ which appears in the action as well as the coupling parameter $g$ are the renormalized values. Also we emphasize that we have chosen to reduce the low frequency part of the field to a single degree of freedom only for simplicity. An inhomogeneous field could be handled, for example, with the techniques presented in Ref. [75].

\section{B. The dissipation and noise kernels: $\gamma$ and $\nu$}

In order to find the noise and dissipation kernels, we use the representations in Eqs. (51) and (52). To compute the averages, observe that the environmental variables can be 
treated as a free field. The details for the evaluation of the correlation $\left\langle\Xi(t) \Xi\left(t^{\prime}\right)\right\rangle$ are reproduced in Appendix B, see Eq. (B1), where we find

$$
\begin{aligned}
\left\langle\Xi(t) \Xi\left(t^{\prime}\right)\right\rangle= & \langle\Xi(t)\rangle\left\langle\Xi\left(t^{\prime}\right)\right\rangle \\
& +\frac{\hbar^{2} g^{2} M^{3}}{8} \int_{p \geqslant M} \frac{d^{3} p}{(2 \pi)^{3}} \frac{e^{-2 i \omega_{p}\left(t-t^{\prime}\right)}}{\omega_{p}^{2}} .
\end{aligned}
$$

One may compute the anticommutator and commutator operator required for the kernels (51) and (52) from this expression. Then comparing with Eqs. (53) and (54), and recalling that the dissipation kernel must be corrected by a factor of $M^{-3}$, in agreement with Eq. (77), we obtain

$$
\begin{aligned}
\hbar \omega \gamma(\omega)= & \frac{\pi \hbar^{2} g^{2}}{4} \int_{p \geqslant M} \frac{d^{3} p}{(2 \pi)^{3} \omega_{p}^{2}}\left[\delta\left(2 \omega_{p}-\omega\right)\right. \\
& \left.-\delta\left(2 \omega_{p}+\omega\right)\right] \\
= & 2 \frac{\pi \hbar^{2} g^{2}}{4} \operatorname{sgn}(\omega) \int_{M(2 \pi)^{3} \omega_{p}^{2}}^{\infty} \delta\left(2 \omega_{p}-|\omega|\right) \\
= & \frac{\hbar^{2} g^{2}}{16 \pi} \operatorname{sgn}(\omega) \sqrt{1-\frac{4 M^{2}}{\omega^{2}}} \theta\left(\omega^{2}-8 M^{2}\right),
\end{aligned}
$$

which leads to

$$
\gamma(\omega)=\frac{\hbar g^{2}}{16 \pi|\omega|} \sqrt{1-\frac{4 M^{2}}{\omega^{2}}} \theta\left(\omega^{2}-8 M^{2}\right) .
$$

With a similar computation, for the noise kernel, we get

$$
\nu(\omega)=\frac{\hbar^{2} g^{2} M^{3}}{32 \pi} \sqrt{1-\frac{4 M^{2}}{\omega^{2}}} \theta\left(\omega^{2}-8 M^{2}\right) .
$$

These kernels are related as required by the zero temperature fluctuation-dissipation theorem, see Eqs. (55). We observe that these are the same as the kernels found in Ref. [13] in a different context. The analysis there substantiates our claim that particle creation, and backreaction thereof, are the main mechanism for dissipation and noise in this model.

\section{Classical trajectories}

Let us recall the basic definitions. The form of the classical potential energy density Eq. (74) suggests writing $\bar{\phi}$ $=\phi_{s} \bar{x}=2 g^{-1} M^{2} \bar{x}$ so that $V(\bar{\phi})=4 g^{-2} M^{6}\left(\bar{x}^{2} / 2-\bar{x}^{3} / 3\right)$. Let us introduce also a dimensionless time $\tau=t M$ and the classical action density for the system, which is defined as $\bar{S}_{s}$ $=M^{3} S_{s}$, reads

$$
\bar{S}_{s}=\frac{4 M^{5}}{g^{2}} \int d \tau\left[\frac{1}{2}\left(\frac{d \bar{x}}{d \tau}\right)^{2}-v(\bar{x})\right],
$$

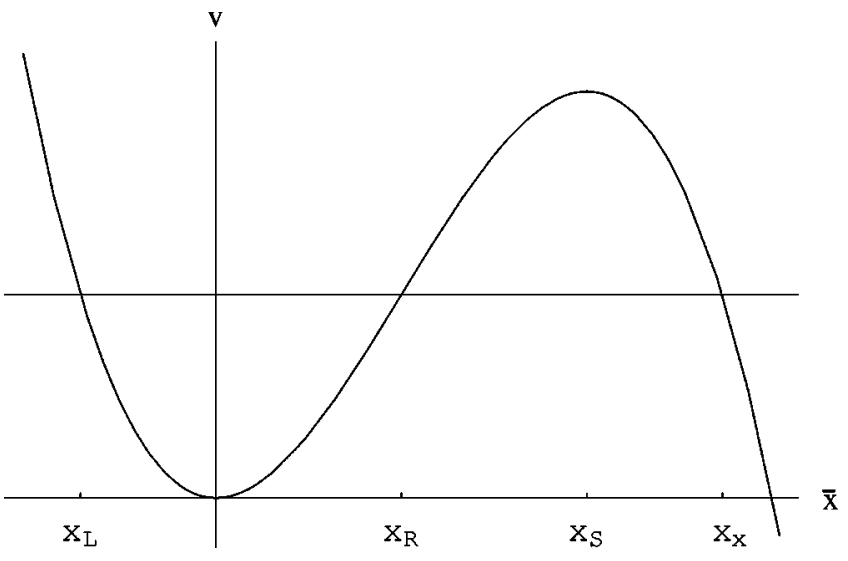

FIG. 1. Plot of the dimensionless potential $v(\bar{x})$, as a function of the dimensionless variable $\bar{x}=\phi / \phi_{s}$. Note that it exhibits a metastable minimum at $\bar{x}=0$ and an unstable maximum at $\bar{x}=x_{s}$, where the value of the potential coincides with the energy $\varepsilon_{s}$ of the separatrix trajectories. The three turning points $x_{L}, x_{R}$ and $x_{\times}$corresponding to any energy $\varepsilon<\varepsilon_{s}$ have also been represented.

where $v(\bar{x})=\bar{x}^{2} / 2-\bar{x}^{3} / 3$. This action density includes dimensionless variables only, the critical point is now $\bar{x}=1$, corresponding to dimensionless energy $\varepsilon_{s}=1 / 6$. Physical energy densities are of course $E=\left(4 M^{6} / g^{2}\right) \varepsilon \equiv 6 E_{s} \varepsilon$.

For any energy $\varepsilon<\varepsilon_{s}$, we shall have three turning points $x_{L}<0<x_{R}<1<x_{\times}$, see Fig. 1. The classical orbits are expressed in terms of Jacobi elliptic functions with modulus $\kappa$ given by

$$
\kappa^{2}=\frac{x_{R}-x_{L}}{x_{\times}-x_{L}},
$$

which satisfies that $\kappa \rightarrow 0$ when $\varepsilon \rightarrow 0$ and $\kappa \rightarrow 1$ when $\varepsilon$ $\rightarrow \varepsilon_{s}$. The actual expression, computed in Appendix C, is

$$
\bar{x}=x_{L}+\left(x_{R}-x_{L}\right) \operatorname{sn}^{2} \lambda \tau,
$$

where $\lambda=\sqrt{\left(x_{\times}-x_{L}\right) / 6}$. The function sn has period $4 K[\kappa]$, and $\mathrm{sn}^{2}$ has half of that, so the dimensionless frequency is $\Omega=\lambda \pi / K$.

The Fourier coefficients of $\bar{x}(\tau)$, for $n \neq 0$, are computed in Appendix D. They are given by

$$
\bar{x}_{n}=-\frac{3 n \Omega^{2}}{\sinh \left(n \Omega K^{\prime} / \lambda\right)} .
$$

\section{The dimensionless Fokker-Planck equation}

Let us first write the noise and dissipation kernels in terms of dimensionless variables. For the system Hamiltonian density, i.e. $\bar{H}_{s}=M^{3} H_{s}$, besides $\bar{x}$ and $\tau(\bar{\phi}$ $\left.=2 g^{-1} M^{2} \bar{x}, \quad t=\tau / M\right)$ we write also $p=2 g^{-1} M^{3} \bar{p}, \quad V$ $=4 g^{-2} M^{6} v(\bar{x})\left[V^{\prime}(\bar{\phi})=2 g^{-1} M^{4} v^{\prime}(\bar{x})\right], \quad$ thereby $\bar{H}_{s}$ $=\left(4 g^{-2} M^{6}\right) \mathbf{h}_{s}$. Note that this transformation is not canonical but preserves the equations of motion, since $p d \bar{\phi}-\bar{H}_{s} d t=\left(4 g^{-2} M^{5}\right)\left(\bar{p} d \bar{x}-\mathbf{h}_{s} d \tau\right)$, see Ref. [76]. The 
action variable also becomes $J=\left(4 g^{-2} M^{5}\right) j$, while the angles are, of course, unchanged. Since the transformation is not canonical, it does not preserve phase volume: $d p d \bar{\phi}=4 g^{-2} M^{5} d \bar{p} d \bar{x}$. Therefore, we ought to define a new distribution function $f(\bar{\phi}, p, t)=\left(g^{2} / 4 M^{5}\right) \bar{f}(\bar{x}, \bar{p}, \tau)$. Then we find $\partial f / \partial t \rightarrow\left(g^{2} / 4 M^{4}\right) \partial \bar{f} / \partial \tau$, and $\left\{\bar{H}_{s}, f\right\}$ $\rightarrow\left(g^{2} / 4 M^{4}\right)\left\{\mathbf{h}_{s}, \bar{f}\right\}_{\bar{x}, \bar{p}}$. Therefore, instead of Eq. (48), we have

$$
\frac{\partial \bar{f}}{\partial \tau}=\left\{\mathbf{h}_{s}, \bar{f}_{\bar{x}, \bar{p}}+\frac{g}{2 M^{4}} \frac{\partial}{\partial \bar{p}}\left[\Gamma(\bar{\phi}, p, t) \bar{f}+\frac{g^{2}}{4 M^{5}}\{N, \bar{f}\}_{\bar{x}, \bar{p}}\right] .\right.
$$

Now let us recall the definitions of $\gamma_{n}$ and $N_{n}$ in Eqs. (58) and (59) and let us introduce the new functions $\omega=M \bar{\omega}, \quad \gamma(\omega)=\left(\hbar g^{2} / M\right) \bar{\gamma}(\bar{\omega}), \quad \gamma_{n}(J)=\hbar g^{2} \bar{\gamma}_{n}(j) \quad$ and $\bar{\phi}_{n}(J)=2 g^{-1} M^{2} \bar{x}_{n}(j)$ to get $\Gamma=2 \hbar g M^{2} \bar{\Gamma}(\bar{x}, \bar{p}, \tau)$. On the other hand, let us call $\nu(\omega)=\hbar^{2} g^{2} M^{3} \bar{\nu}(\bar{\omega}), N_{n}$ $=\hbar^{2} g^{2} M^{3} \bar{N}_{n}$ and $N=2 \hbar^{2} g M^{5} \bar{N}$ and we finally obtain the following dimensionless form of the Fokker-Planck equation

$$
\frac{\partial \bar{f}}{\partial \tau}=\left\{\mathbf{h}_{s}, \bar{f}\right\}_{\bar{x}, \bar{p}}+\frac{4}{\beta} \frac{\partial}{\partial \bar{p}}\left[\bar{\Gamma}(\bar{\phi}, p, t) \bar{f}+\frac{1}{\beta}\{\bar{N}, \bar{f}\}_{\bar{x}, \bar{p}}\right],
$$

where $\beta=4 M^{2} /\left(\hbar g^{2}\right)$. In dimensionless variables, the energy scale for the false vacuum is $1 /(2 \beta)$.

We may now proceed to write down the angle averaged Fokker-Planck equation (60) in dimensionless variables. This takes the form

$$
\frac{\partial \bar{f}}{\partial \tau}=\frac{4}{\beta} \frac{d}{d j}\left\{\frac{\overline{\mathbf{N}}}{\beta \bar{\Omega}} \frac{d \bar{f}}{d j}+\overline{\mathbf{D}} \bar{f}\right\},
$$

where $\quad \overline{\mathbf{D}}=(\bar{\Omega} / 2) \Sigma_{n}\left|\bar{x}_{n}(j)\right|^{2} n^{2} \bar{\gamma}[n \bar{\Omega}] \quad$ and $\quad \overline{\mathbf{N}}$ $=(\bar{\Omega} / 2) \Sigma_{n}\left|\bar{x}_{n}(j)\right|^{2} n^{2} \bar{\nu}[n \bar{\Omega}]$, and we have also introduced the dimensionless $\bar{\Omega}=\Omega / M$. Using the explicit form of the Fourier coefficients Eq. (84) and the expressions (79) and (80) for the dissipation and noise kernels, $\gamma$ and $\nu$, we have explicitly

$$
\begin{aligned}
& \overline{\mathbf{D}}=\frac{9 \bar{\Omega}}{16 \pi} \sum_{n>0} \frac{(n \bar{\Omega})^{3}}{\sinh ^{2}\left(n \bar{\Omega} K^{\prime} / \lambda\right)} \sqrt{1-\frac{4}{n^{2} \bar{\Omega}^{2}}} \theta\left(n^{2} \bar{\Omega}^{2}-8\right), \\
& \overline{\mathbf{N}}=\frac{9 \bar{\Omega}}{32 \pi} \sum_{n>0} \frac{(n \bar{\Omega})^{4}}{\sinh ^{2}\left(n \bar{\Omega} K^{\prime} / \lambda\right)} \sqrt{1-\frac{4}{n^{2} \bar{\Omega}^{2}}} \theta\left(n^{2} \bar{\Omega}^{2}-8\right) .
\end{aligned}
$$

There are two relevant limiting cases. The first limit corresponds to the separatrix energy, that is, when $\varepsilon \rightarrow \varepsilon_{s}, \bar{\Omega}$ $\rightarrow 0, \lambda \rightarrow 1 / 2, K^{\prime} \rightarrow \pi / 2$. We may write $\xi=n \bar{\Omega}$ and $\Sigma_{n}$ $\sim(1 / \bar{\Omega}) \int d \xi$ in the previous equations. Then after numerical integration we obtain

$$
\begin{aligned}
& \overline{\mathbf{D}} \equiv \overline{\mathbf{D}}_{s} \sim \frac{9}{16 \pi} 2.441 \ldots \times 10^{-7}, \\
& \overline{\mathbf{N}} \equiv \overline{\mathbf{N}}_{s} \sim \frac{9}{32 \pi} 7.381 \ldots \times 10^{-7},
\end{aligned}
$$

which remain finite. The second limit corresponds to the bottom of the potential, that is, when $\varepsilon \rightarrow 0, \bar{\Omega} \rightarrow 1, \lambda \rightarrow 1 / 2$. Then $\quad K^{\prime} \sim(1 / 2) \ln \left(16 \kappa^{-2}\right)=(1 / 2) \ln \left[24\left(x_{R}-x_{L}\right)^{-1}\right]$ $\sim(1 / 4) \ln (72 / \varepsilon)$, see Ref. [77], formula 8.113.3, and $\sinh ^{-2}\left(n \bar{\Omega} K^{\prime} / \lambda\right) \sim 4 \exp \left(-4 n K^{\prime}\right) \sim 4(\varepsilon / 72)^{n}$ and the expressions for $\overline{\mathbf{D}}$ and $\overline{\mathbf{N}}$ take the values

$$
\begin{aligned}
& \overline{\mathbf{D}} \sim \frac{9}{4 \pi} \sum_{n=3} n^{3}\left(\frac{\varepsilon}{72}\right)^{n} \sqrt{1-\frac{4}{n^{2}}} \sim b \varepsilon^{3}, \\
& \overline{\mathbf{N}} \sim \frac{9}{8 \pi} \sum_{n=3} n^{4}\left(\frac{\varepsilon}{72}\right)^{n} \sqrt{1-\frac{4}{n^{2}}} \sim a \varepsilon^{3},
\end{aligned}
$$

where $a$ and $b$ are numerical coefficients that can be read from these expressions. Note that the rapid decay of these functions as $\varepsilon \rightarrow 0$ is due to the presence of a threshold, encoded in the theta functions of Eqs. (79) and (80), which enforce that $n^{2} \bar{\Omega}^{2}>8$. At finite temperature, where the selfenergy of the fluctuations remains complex even on-shell (when higher loops are included, of course), $\overline{\mathbf{D}}$ and $\overline{\mathbf{N}}$ fall like $\varepsilon$, as in Kramers' original analysis [7].

\section{ENVIRONMENT INDUCED DECAY RATES}

We may now conclude our task of finding the decay rates as described by the Fokker-Planck equation. In agreement with our weak dissipation assumptions we shall assume that we may average over angles, and restrict our analysis to the Fokker-Planck equation (87); a related analysis of angledependent solutions is given in Appendix 6 of Ref. [63]. Since the equation is linear in the Wigner function, it is convenient to first seek the normal modes, namely, solutions with a simple (exponential) dependence on time. The desired solution will then be reconstructed as a superposition of normal modes $[69,78]$.

Assuming then that $\bar{f}=e^{-r \tau} F(j)$, we get from Eq. (87) the time independent equation

$$
L F+r F=0, \quad L=\frac{4}{\beta} \frac{d}{d j}\left(\frac{\overline{\mathbf{N}}}{\beta \bar{\Omega}} \frac{d}{d j}+\overline{\mathbf{D}}\right) .
$$

This equation may be written as a conservation equation $L F=d \Phi / d j$, where $\Phi$ is the flux defined by

$$
\bar{\Phi}=\frac{4}{\beta}\left(\frac{\overline{\mathbf{N}}}{\beta \bar{\Omega}} \frac{d F}{d j}+\overline{\mathbf{D}} F\right) .
$$

Observe that for any $\varepsilon>\varepsilon^{\prime}>0$ we have the following relationship between $F, \Phi$ and $r$ : 


$$
r=\frac{\bar{\Phi}(\varepsilon)-\bar{\Phi}\left(\varepsilon^{\prime}\right)}{\int_{\varepsilon^{\prime}}^{\varepsilon} F d j}
$$

Let us now introduce the unnormalized equilibrium solution $F_{B}=\exp \left(-\beta \int d j \Omega \bar{\Omega} / \overline{\mathbf{D}}\right)$, then Eq. (92) can be written as

$$
\frac{d}{d j}\left[\rho^{2} \frac{d}{d j}\left(\frac{F}{F_{B}}\right)\right]+r F=0,
$$

where $\rho^{2}(j)=4 \overline{\mathbf{N}} F_{B} /\left(\bar{\Omega} \beta^{2}\right)$. Multiplying by $F / F_{B}$ and integrating we see that $r$ must be non-negative, as expected. From the mathematical point of view, this is an eigenvalue problem of the Sturm-Liouville type, and we may handle it in the usual way [79]. Let us begin by analyzing the solutions with $r \neq 0$, which are the decaying solutions.

\section{A. Decaying solutions}

Let us seek a solution $F_{K}$ of Eq. (95) with nonzero $r$. Since later on we shall be interested in the long time behavior of solutions, we may focus on the range of small values of $r$. For concreteness, let us assume $r \beta^{3} / 3 a \ll 1$. Let us introduce $h$ by $F_{K}=(h / \rho) F_{B}=(\beta h / 2) \sqrt{\bar{\Omega} F_{B} / \overline{\mathbf{N}}}$ and write Eq. (95) and the flux as

$$
\begin{gathered}
\frac{d}{d j}\left[\rho^{2} \frac{d}{d j}\left(\frac{h}{\rho}\right)\right]+\frac{r \bar{\Omega} \beta^{2}}{4 \overline{\mathbf{N}}} \rho h=0, \\
\bar{\Phi}=-\rho^{2} \frac{d}{d j}\left(\frac{h}{\rho}\right)=-\left(\rho h^{\prime}-h \rho^{\prime}\right) .
\end{gathered}
$$

Expanding the second derivatives $d_{j}\left[\rho^{2} d_{j}(h / \rho)\right]=d_{j}\left(\rho h^{\prime}\right.$ $\left.-h \rho^{\prime}\right)=\rho h^{\prime \prime}-h \rho^{\prime \prime}$, where $d_{j}$ stands for the derivative with respect to $j$, we obtain

$$
h^{\prime \prime}+\left(\frac{r \bar{\Omega} \beta^{2}}{4 \overline{\mathbf{N}}}-\frac{\rho^{\prime \prime}}{\rho}\right) h=0 .
$$

We have two regimes in this equation: for large $\varepsilon$ (but not exponentially close to $\left.\varepsilon_{s}=1 / 6\right), \rho$ is dominated by $F_{B}$, and $\rho^{\prime \prime} / \rho \sim \beta^{2} \bar{\Omega}^{2} \overline{\mathbf{D}}^{2} /\left(4 \overline{\mathbf{N}}^{2}\right)$ dominates the $r$ term, which is negligible. For $\varepsilon \rightarrow 0$, on the other hand, $\rho \sim \varepsilon^{3 / 2}, \rho^{\prime \prime} / \rho$ $\sim(3 / 4) \varepsilon^{-2}$, and the $r$ term is the leading term. The transition occurs for $\varepsilon=\varepsilon^{*} \sim r \beta^{2} /(3 a)$, which by assumption is much smaller than the energy scale $1 / 2 \beta$ typical of the false vacuum.

For large $\varepsilon$, the equation $h^{\prime \prime}-\left(\rho^{\prime \prime} / \rho\right) h=0$ admits of course the solution $h=\rho$, which gives back the equilibrium solution, i.e. $F_{K}=F_{B}$. To find the second solution we may write $h=K_{r} \rho \sigma$ (here $K_{r}$ is a constant) to get $\sigma^{\prime \prime} / \sigma^{\prime}=$ $-2 \rho^{\prime} / \rho$ which implies $\sigma^{\prime}=-\rho^{-2}$. This second solution corresponds to a constant flux $\Phi=K_{r}$, and also is the negative exponential WKB solution. For small $\varepsilon$, the equation $h^{\prime \prime}+\left(r \beta^{2} / 4 a \varepsilon^{3}\right) h=0$ has solutions

$$
h=C_{r} \sqrt{\varepsilon} Z_{1}\left[\frac{k_{r}}{\sqrt{\varepsilon}}\right],
$$

where $k_{r}^{2}=r \beta^{2} / a, Z$ is a Bessel function and $C_{r}$ is a constant (observe that all solutions are bounded). As $\varepsilon \rightarrow 0, F$ $\sim \varepsilon^{-3 / 4} \cos \left(k \varepsilon^{-1 / 2}\right)$ but $\bar{\Phi} \sim \varepsilon^{3 / 4} \sin \left(k \varepsilon^{-1 / 2}\right) \rightarrow 0$.

Note that we obtain solutions for arbitrarily small values of $r$. This behavior, which is unlike that found by Kramers [7], reflects the existence of a threshold for dissipation: at arbitrarily low energies, the motion is essentially harmonic, there is no dissipation, the fluctuations switch off (in agreement with the fluctuation-dissipation theorem) and the system requires an infinite time to climb out of the potential well.

\section{B. Hilbert space structure and normalization}

The structure of Eq. (95) suggests introducing an inner product

$$
\langle f, g\rangle=\int_{0}^{j_{s}} \frac{d j}{F_{B}} f^{*} g,
$$

where the star means complex conjugation. Then, if $F_{r}$ and $F_{s}$ are the solutions corresponding to eigenvalues $r$ and $s$, we can write $(r-s)\left\langle F_{s}, F_{r}\right\rangle=-\int_{0}^{j_{s}} d j F_{B}^{-1}\left[F_{s}\left(L F_{r}\right)\right.$ $\left.-\left(L F_{s}\right) F_{r}\right]=-\left.F_{B}^{-1}\left[F_{s} \Phi_{r}-\Phi_{s} F_{r}\right]\right|_{0} ^{j_{s}}$. Imposing the boundary conditions $F_{r}\left(j_{s}\right)=0$, we get rid of the contribution from the upper limit. In the lower limit, we may use the asymptotic form of the Bessel functions to get $\left[F_{s} \Phi_{r}\right.$ $\left.-\Phi_{s} F_{r}\right](\varepsilon) \sim 2 \pi^{-1} C_{r} C_{s} k_{r}^{-1 / 2} k_{s}^{-1 / 2} \sin \left[\left(k_{r}-k_{s}\right) \varepsilon^{-1 / 2}\right] \quad$ which converges (weakly) to zero. It is therefore natural to adopt the continuum normalization prescription

$$
\left\langle F_{s}, F_{r}\right\rangle \equiv \delta(r-s)
$$

As in Landau and Lifschitz' analysis of the WKB wave functions [5], this singular behavior is caused by the oscillations as $\varepsilon \rightarrow 0$. More precisely

$$
\begin{aligned}
\int_{0} \operatorname{dj} F_{B}^{-1} F_{r} F_{s} \sim & \pi^{-1} C_{r} C_{s} k_{r}^{-1 / 2} k_{s}^{-1 / 2} \int_{0} d \varepsilon \varepsilon^{-3 / 2} \\
& \times \cos \left[\left(k_{r}-k_{s}\right) \varepsilon^{-1 / 2}\right] \sim 4 C_{r}^{2} k_{r}^{-1} \delta\left(k_{r}-k_{s}\right) \\
& \sim 8 a C_{r}^{2} \beta^{-2} \delta(r-s),
\end{aligned}
$$

where the integral upper limit is anything. We thus find the constants $C_{r}$

$$
C_{r} \sim \beta / \sqrt{8 a} .
$$

To find the constants $K_{r}$ we should match our solutions across the transition point at $\varepsilon^{*}$. Without getting into details, see Ref. [80], it is clear that in order of magnitude 


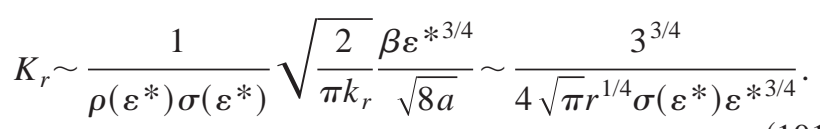

To find $\sigma\left(\varepsilon^{*}\right)$, we recall that according to our boundary conditions $\sigma\left(j_{s}\right)=0$. Therefore $\sigma\left(\varepsilon^{*}\right)=\int_{\varepsilon^{*}}^{\varepsilon_{s}} d j \rho^{-2}(j)$. Let us split the range of integration in the two segments $\left[\varepsilon^{*}, 1 / 2 \beta\right]$ and $[1 / 2 \beta, \varepsilon]$. The contribution $I_{I I}$ from the second segment is dominated by the exponential growth of $F_{B}^{-1}$ near the separatrix, thus $I_{I I} \sim F_{B}(1 / 2 \beta) F_{B}^{-1}\left(\varepsilon_{s}\right)$ $\sim \Delta^{\prime}-1 \exp \left(\beta \overline{\mathbf{D}}_{s} / 6 \overline{\mathbf{N}}_{s}\right)$ where $\Delta^{\prime} \sim O(1)$. In the first segment, we find

$$
\rho^{-2}=\beta^{2} /\left(4 a \varepsilon^{3}\right),
$$

so it contributes as

$$
\begin{aligned}
I_{I} & \sim(1 / 8) \beta^{2} a^{-1}\left[\left(\varepsilon^{*}\right)^{-2}-(1 / 4) \beta^{-2}\right] \\
& \sim(1 / 8) \beta^{2} a^{-1}\left[9 a^{2} \beta^{-4} r^{-2}-(1 / 4) \beta^{-2}\right] \\
& \sim(9 / 8) a \beta^{-2}\left[r^{-2}-(1 / 36) \beta^{2} a^{-2}\right] .
\end{aligned}
$$

Adding both contributions, and keeping only the leading terms, we find

$$
\sigma\left(\varepsilon^{*}\right)=\frac{9 a}{8 \beta^{2}}\left(\frac{1}{r^{2}}+\frac{1}{\lambda^{2}}\right),
$$

where $\lambda \sim \widetilde{\Delta} \exp \left(-\beta \overline{\mathbf{D}}_{s} / 12 \overline{\mathbf{N}}_{s}\right)$ with $\widetilde{\Delta} \sim O(1)$. So finally we have

$$
K_{r} \sim K \frac{\lambda^{2} r}{r^{2}+\lambda^{2}},
$$

where $K$ is an $r$-independent constant.

\section{Decay of the false vacuum}

After all this work, the dynamical problem is now trivial. We are interested in a time-dependent solution with an initial condition similar to the Wigner function of the false vacuum, $F \equiv F_{F V} \sim 2 \beta \exp (-2 \beta \varepsilon)[81]$. The solution is

$$
F(j, t)=\int_{0}^{\infty} d r e^{-r t} c_{r} F_{r}(j)
$$

where $c_{r}=\int_{0}^{j_{s}} d j F_{B}^{-1} F_{r} F_{F V}$. Fortunately, we are interested in the range of small $r$, where $F_{r}$ peaks at values much smaller than $1 / 2 \beta$. The only feature of $F_{F V}$ and $F_{B}$ that we really need is that they are smooth there. Thus, $c_{r}$ $\sim F_{F V}(0) F_{B}^{-1}(0) \int_{0}^{j_{s}} d j F_{r}=2 \beta K_{r} / r$, [cf. Eq. (94)]. Finally

$$
F(j, t)=2 \beta K \lambda^{2} \int_{0}^{\infty} d r \frac{e^{-r t}}{r^{2}+\lambda^{2}} F_{r}(j)
$$

To find the persistence probability, $P(t)$, we integrate $F(j, t)$ over the potential well. Thus after using the previous result for $\int_{0}^{j_{s}} d j F_{r}(j)$ and the value of $K_{r}$ from (102) we obtain

$$
P(t) \sim 2 \beta K^{2} \int_{0}^{\infty} d r \frac{\lambda^{4} e^{-r t}}{\left[r^{2}+\lambda^{2}\right]^{2}} .
$$

The analytic expression for this integral is given in Ref. [77], formula 3.355.1. When $\lambda t \sim 1$ it can be approximated with an integral, the best fit is obtained when $P(t)$ $\sim \exp (-0.4 \lambda t)$. For larger times, we have a crossover from exponential to power law $1 / t$ decay, as expected from quantum mechanics (cf. [15]). To summarize, we have proven that the Fokker-Planck equation leads to an exponential decay of the false vacuum, with a decay rate $t_{d}^{-1} \sim \lambda$ :

$$
t_{d}^{-1} \sim \Delta \exp \left(\frac{-\mathcal{D}_{s}}{12 \mathcal{N}_{s}} \beta\right),
$$

with $\Delta \sim O(1)$. This equation may be compared to Eq. (1), here $\beta=4 M^{2} /\left(\hbar g^{2}\right)$, as defined in Eq. (86), and the ratio $\mathcal{D}_{s} / \mathcal{N}_{s}=\overline{\mathbf{D}}_{s} / \overline{\mathbf{N}}_{s}$ where $\overline{\mathbf{D}}_{s}$ and $\overline{\mathbf{N}}_{s}$ are given in (91); see Eqs. (63), (64) and (87) for the definitions of the functions involved.

\section{Comparing with the instanton method}

Let us conclude by comparing the rate estimate of Eq. (103) with the estimate derived from the instanton method. The Euclidean action for our model is

$$
S_{E}[\Phi]=\int d^{4} x\left(\frac{1}{2} \delta^{\mu \nu} \partial_{\mu} \Phi \partial_{\nu} \Phi+\frac{1}{2} M^{2} \Phi^{2}-\frac{1}{6} g \Phi^{3}\right),
$$

where $\delta^{\mu \nu}$ stands for the Euclidean metric. We are interested in $S O(4)$ symmetric instantons, which depend on all four Euclidean coordinates but only through the Euclidean radius $\rho=\left(x^{2}+y^{2}+z^{2}+t_{E}^{2}\right)^{1 / 2}$, where $t_{E}$ is the Euclidean time. Let us scale $\Phi=\phi_{s} f(M \rho)$ to get the Euclidean action (in $d$ dimensions) $S_{d}=(4 \pi / 3) \hbar \beta I_{d}$, where

$$
I_{d}=\int d \rho \rho^{d-1}\left[\frac{1}{2}\left(f^{\prime}\right)^{2}+\frac{1}{2} f^{2}-\frac{1}{3} f^{3}\right] .
$$

Of course, computing the four-dimensional $(d=4)$ instanton is not simple, but we may approximate $I_{4}$ by $I_{1}$, in which case we may use the simpler one-dimensional formula

$$
S=2 \int_{0}^{3 / 2} d x \sqrt{2 v(x)}=2 \int d x \sqrt{x^{2}-\frac{2}{3} x^{3}}=\frac{6}{5} .
$$

All in all, the instanton prediction is $\ln t_{d}^{-1} \sim-1.2 \ldots \times \beta$. On the other hand, the noise induced prediction, Eq. (103), is $\ln t_{d}^{-1} \sim \ln \lambda \sim\left(-\overline{\mathbf{D}}_{s} / 12 \overline{\mathbf{N}}_{s}\right) \beta \sim-0.05 \ldots \times \beta$. So in this simple case the noise induced contribution dominates over the instanton contribution. 


\section{CONCLUSIONS}

In this paper we have studied the contribution to vacuum decay in field theory as a consequence of the interaction between the long and short-wavelength modes. We have seen that the dynamics of the long-wavelength modes becomes diffusive in its interaction with the short wavelength modes. On the one hand, there is dissipation of the long-wavelength modes due to the excitation of the short-wavelength sector, and in turn, that latter sector induces fluctuations into the first sector. As a result there is a significant contribution to the total decay rate due to activation, even at zero temperature.

A few remarks are in order. What we have shown is that if we consider the field initially in a metastable phase in a region of size $M^{-1}$ there is a probability per unit volume and unit time of decay to a stable phase given by Eq. (103). Of course, the size of the bubble formed is of order $M^{-1}$, in our calculation this size is fixed. We cannot consider smaller size regions because we could not have neglected the gradient terms of the system in front of the mass terms, also we could not have considered larger size regions because the environment modes would become unstable, their evolution would become nonlinear and our perturbative treatment of the influence functional would break down. However, the critical bubble size that one obtains in first order phase transition in statistical physics or in field theory using instanton methods is also of the order of $M^{-1}$. This, in our opinion, makes this computation of interest, since one expects that once the critical bubble is formed it will evolve in the usual way; see for instance Ref. [9]. Had our calculation involved a size smaller than the critical size then the bubbles formed could not grow and would collapse, as then the energy of the bubble wall would overcome the energy difference between the metastable phase and the bubble interior.

Another relevant point concerns the energy balance in the activation mechanism of vacuum decay described here which involves noise and dissipation. In Appendix E we show that the average power exchanged between system and environment is zero: if some trajectories gain power through noise, some others lose power through dissipation. Of course, the balance is only statistical, but we must stress that this gain and loss process would go on even if there were no separatrix and the system were in equilibrium. The only reason why the system does not equilibrate in our problem is that we remove those particles that reach the separatrix, as demanded by our boundary condition there. We should say that the system equilibrates but we assume that the true vacuum is very much deeper than the false vacuum, so the equilibrium distribution vanishes inside the potential well. Note that this is an expected result in vacuum bubble formation. Once the critical bubble is formed the energy released in the conversion from false to true vacuum is converted into energy of the growing bubble wall, so that the energy balance is still zero. This last aspect however cannot be studied with the present analysis.

\section{ACKNOWLEDGMENTS}

We are grateful to Daniel Arteaga, Leticia Cugliandolo, Jaume Garriga, Rodolfo Id Betan, Bei-Lok Hu, Ted Jacobson and Jorge Kurchan for many interesting discussions. This work has been partially supported by Fundación Antorchas under grant A-13622/1-21. E.C. acknowledges support from Universidad de Buenos Aires, CONICET, Fundación Antorchas and the ANPCYT through Project PICT99 03-05229. A.R. and E.V. have also been supported by the CICYT Research Project No. AEN98-0431, A.R. also acknowledges partial support by a grant from the Generalitat de Catalunya, and E.V. also acknowledges support from the Spanish Ministry of Education under the FPU grant PR2000-0181 and the University of Maryland for hospitality.

\section{APPENDIX A}

Our problem is to compute an expression like

$$
\int d t^{\prime} N\left(t-t^{\prime}\right)\left\langle\frac{\delta}{\delta \xi\left(t^{\prime}\right)} R[X(t), p(t)]\right\rangle,
$$

where $R$ is an arbitrary functional of $\xi(t)$ and $\langle\ldots\rangle$ denotes expectation value with respect to $P_{Q}[\xi ; t)$. Here, for simplicity, we will assume also that $P_{Q}$ is independent of $X$ as in the case of the cubic potential. In our case, this leads to

$$
\begin{aligned}
\widetilde{\Phi}[\xi]= & -\hbar \int d t^{\prime} N\left(t-t^{\prime}\right)\left[\frac{\partial}{\partial X}\left\langle\frac{\delta X(t)}{\delta \xi(\tau)} \delta_{X} \delta_{p}\right\rangle\right. \\
& \left.+\frac{\partial}{\partial p}\left\langle\frac{\delta p(t)}{\delta \xi(\tau)} \delta_{X} \delta_{p}\right\rangle\right]
\end{aligned}
$$

where $\delta_{X} \equiv \delta(X(t)-X)$ and $\delta_{p} \equiv \delta(p(t)-p)$.

Within the "reduction of order" procedure, we substitute $\partial X(\tau) / \partial X(t)$ and $\partial X(\tau) / \partial p(t)$ for $\delta X(t) / \delta \xi(\tau)$ and $\delta p(t) / \delta \xi(\tau)$, where the variations are understood in the sense of the result of coupling a stochastic source to the classical equations of motion. The argument runs as follows: we know that $\delta X\left(\tau^{+}\right) / \delta \xi(\tau)=0$ and $\delta p\left(\tau^{+}\right) / \delta \xi(\tau)=1$. On the other hand

$$
\delta_{\xi(\tau)} X\left(\tau^{+}\right)=\partial_{X(t)} X\left(\tau^{+}\right) \delta_{\xi(\tau)} X(t)+\partial_{p(t)} X\left(\tau^{+}\right) \delta_{\xi(\tau)} p(t)
$$

and

$$
\delta_{\xi(\tau)} p\left(\tau^{+}\right)=\partial_{X(t)} p\left(\tau^{+}\right) \delta_{\xi(\tau)} X(t)+\partial_{p(t)} p\left(\tau^{+}\right) \delta_{\xi(\tau)} p(t) .
$$

By Liouville's theorem the determinant of this two by two system is 1 , so indeed

$$
\frac{\delta X(t)}{\delta \xi(\tau)}=-\frac{\partial X\left(\tau^{+}\right)}{\partial p(t)}, \quad \frac{\delta p(t)}{\delta \xi(\tau)}=\frac{\partial X\left(\tau^{+}\right)}{\partial X(t)} .
$$

The right-hand sides of these equations are continuous, so we may omit the superscript. Furthermore

$$
\frac{\partial X(\tau)}{\partial p(t)}=\{X(t), X(\tau)\}, \quad \frac{\partial X(\tau)}{\partial X(t)}=-\{p(t), X(\tau)\},
$$

and computing the Poisson brackets in terms of the canonical variables $\theta$ and $J$ we arrive at 


$$
\begin{aligned}
\widetilde{\Phi}[\xi]= & \hbar \int d t^{\prime} N\left(t-t^{\prime}\right)\left[\frac{\partial}{\partial X}\left[\left\{X(t), X\left(t^{\prime}\right)\right\} f\right]\right. \\
& \left.+\frac{\partial}{\partial p}\left[\left\{p(t), X\left(t^{\prime}\right)\right\} f\right]\right] \\
= & \frac{\partial}{\partial X}[\{X(t), N(J, \theta)\} f]+\frac{\partial}{\partial p}[\{p(t), N(J, \theta)\} f],
\end{aligned}
$$

where we have defined $N(J, \theta) \equiv \hbar \int d t^{\prime} N\left(t-t^{\prime}\right) X\left(t^{\prime}\right)$. Applying the reduction of order procedure by substituting $X\left(t^{\prime}\right)$ by the Fourier expression (56) and using Eq. (54) the function $N(J, \theta)$ is written as,

$$
N(J, \theta)=\sum_{n} X_{n}(J) e^{i n[\theta+\Omega(J) t]} N_{n}(J)
$$

where $N_{n}(J)$ is given by Eq. (59). We may simplify further, by observing that $\{X,\{p, N\}\}-\{p,\{X, N\}\}=-\{N,\{X, p\}\}$ $=0$ by the Jacobi identity and the fact that $\{X, p\}=1$, then by further manipulation of the Poisson bracket terms we finally get

$$
\begin{aligned}
\widetilde{\Phi}[\xi] & =\{X,\{p, N(J, \theta)\} f\}-\{p,\{X, N(J, \theta)\} f\} \\
& =\{p, N(J, \theta)\}\{X, f\}-\{X, N(J, \theta)\}\{p, f\} \\
& =\left[-\frac{\partial p}{\partial J} \frac{\partial X}{\partial \theta}+\frac{\partial X}{\partial J} \frac{\partial p}{\partial \theta}\right]\left[\frac{\partial N}{\partial \theta} \frac{\partial f}{\partial J}-\frac{\partial N}{\partial J} \frac{\partial f}{\partial \theta}\right] \\
& =-\{N, f\} .
\end{aligned}
$$

\section{APPENDIX B}

We first expand the environmental variables in creation and annihilation operators

$$
\varphi_{\vec{k}}=\sqrt{\frac{\hbar}{2 \omega_{k}}}\left[a_{k} e^{-i \omega_{k} t}+a_{-k}^{\dagger} e^{i \omega_{k} t}\right],
$$

where $\omega_{k}^{2}=k^{2}+M^{2}$. Next, recalling the definition of $\Xi$ given in Eq. (75) we may write

$$
\begin{aligned}
\Xi(t)= & \frac{1}{2} g M^{3} \int_{M^{-3}} d^{3} x \int \frac{d^{3} k}{(2 \pi)^{3}} e^{i \vec{k} \cdot \vec{x}} \\
& \times \int_{p, k-p \geqslant M} \frac{d^{3} p}{(2 \pi)^{3}} \varphi_{\vec{p}} \varphi_{\vec{k}-\vec{p}} .
\end{aligned}
$$

To perform the integral over $x$ we introduce as in Sec. IV the function $\rho(k / M)=M^{3} \int_{M^{-3}} d^{3} x \exp (i \vec{k} \cdot \vec{x})$, which satisfies $\rho(0)=1$. Then by direct substitution we can write the correlation $\left\langle\Xi(t) \Xi\left(t^{\prime}\right)\right\rangle$ as

$$
\begin{aligned}
\left\langle\Xi(t) \Xi\left(t^{\prime}\right)\right\rangle= & \langle\Xi(t)\rangle\left\langle\Xi\left(t^{\prime}\right)\right\rangle \\
& +\frac{\hbar^{2} g^{2}}{16} \int \frac{d^{3} k}{(2 \pi)^{3}} \frac{d^{3} k^{\prime}}{(2 \pi)^{3}} \rho\left(\frac{k}{M}\right) \rho\left(\frac{k^{\prime}}{M}\right) \\
& \times \int_{p, k-p \geqslant M} \frac{d^{3} p}{(2 \pi)^{3}} \int_{p^{\prime}, k^{\prime}-p^{\prime} \geqslant M} \frac{d^{3} p^{\prime}}{(2 \pi)^{3}} \\
& \times \frac{e^{-i \omega_{p} t} e^{i \omega_{p^{\prime}-k^{\prime} t^{\prime}}} e^{-i \omega_{k-p^{t}} e^{i \omega_{p^{\prime}} t^{\prime}}}}{\sqrt{\omega_{p} \omega_{p-k^{\prime}} \omega_{p^{\prime}} \omega_{p^{\prime}-k^{\prime}}}} \\
& \times\left\langle a_{p} a_{k-p} a_{-p^{\prime}}^{\dagger} a_{p^{\prime}-k^{\prime}}^{\dagger} .\right.
\end{aligned}
$$

Now the vacuum expectation value in the last equation can be written as

$$
\begin{aligned}
\left\langle a_{p} a_{k-p} a_{-p^{\prime}}^{\dagger} a_{p^{\prime}-k^{\prime}}^{\dagger}\right\rangle= & (2 \pi)^{6} \delta^{(3)}\left(\vec{k}+\vec{k}^{\prime}\right)\left\{\delta^{(3)}\left(\vec{k}-\vec{p}+\vec{p}^{\prime}\right)\right. \\
& \left.+\delta^{(3)}\left(\vec{p}+\vec{p}^{\prime}\right)\right\},
\end{aligned}
$$

and since in any case we deal with values of $k$ and $k^{\prime}$ much lower than typical values of $p$ and $p^{\prime}$, we get

$$
\begin{aligned}
&\left\langle\Xi(t) \Xi\left(t^{\prime}\right)\right\rangle=\langle\Xi(t)\rangle\left\langle\Xi\left(t^{\prime}\right)\right\rangle+\frac{\hbar^{2} g^{2}}{8} \int \frac{d^{3} k}{(2 \pi)^{3}} \rho^{2}\left(\frac{k}{M}\right) \\
& \times \int_{p \geqslant M} \frac{d^{3} p}{(2 \pi)^{3}} \frac{e^{-2 i \omega_{p}\left(t-t^{\prime}\right)}}{\omega_{p}^{2}} .
\end{aligned}
$$

To obtain Eq. (78), observe that $\int d^{3} k \rho^{2}(k / M)=(2 \pi)^{3} M^{3}$.

\section{APPENDIX C}

Let us write the integrand in the action density (81) as

$$
\varepsilon-v(\bar{x})=\frac{1}{3}\left(\bar{x}-x_{L}\right)\left(\bar{x}-x_{R}\right)\left(\bar{x}-x_{\times}\right) .
$$

It can be rearranged as $\varepsilon-v(\bar{x})=(1 / 3)\left(x_{\times}-\bar{x}\right)\left\{(1 / 4)\left(x_{R}\right.\right.$ $\left.\left.-x_{L}\right)^{2}-\left[\bar{x}-(1 / 2)\left(x_{R}+x_{L}\right)\right]^{2}\right\}$, which suggests writing $\bar{x}$ $=(1 / 2)\left(x_{R}+x_{L}\right)-(1 / 2)\left(x_{R}-x_{L}\right) \cos (2 \varphi)=x_{L}+\left(x_{R}-x_{L}\right) \sin ^{2} \varphi$, and thus

$$
\varepsilon-v(\bar{x})=\frac{1}{12}\left(x_{\times}-x_{L}\right)\left(x_{R}-x_{L}\right)^{2} \sin ^{2} 2 \varphi\left(1-\kappa^{2} \sin ^{2} \varphi\right),
$$

where $\kappa$ is defined in Eq. (82).

The equation for a classical trajectory is $\tau=\int d \bar{x} \bar{p}^{-1}$ $=\int d \bar{x}\{2[\varepsilon-v(\bar{x})]\}^{-1 / 2}$ and since $d \bar{x}=\left(x_{R}-x_{L}\right) \sin (2 \varphi) d \varphi$ we have

$$
\tau=\sqrt{\frac{6}{x_{\times}-x_{L}}} \int_{0}^{\varphi} \frac{d \varphi^{\prime}}{\sqrt{1-\kappa^{2} \sin ^{2} \varphi^{\prime}}} .
$$


When $\varphi$ goes from 0 to $\pi / 2$, the point describes a half orbit. So the period $T$ is twice the result from Eq. (C3) when $\varphi$ $=\pi / 2$, and one can check that when $\varepsilon \rightarrow 0, T \rightarrow 2 \pi$, as it should.

Using now the identities 16.1.3, 4 and 5 from Ref. [83], we get the desired formula (83). Observe that as $\varepsilon$ $\rightarrow 0, \quad \operatorname{sn} u \sim \sin u, \quad \lambda \sim 1 / 2, \quad$ and $\bar{x}=x_{L}+\left(x_{R}-x_{L}\right) \operatorname{sn}^{2}(\tau / 2)$ $\sim(1 / 2)\left(x_{R}+x_{L}\right)-(1 / 2)\left(x_{R}-x_{L}\right) \cos \tau$ which corresponds to the harmonic trajectory, as expected near the bottom of the potential.

\section{APPENDIX D}

Here we shall borrow an argument from Whittaker and Watson [84]. The Fourier coefficients of the classical trajectory are

$$
\begin{aligned}
\bar{x}_{n} & =\frac{1}{T} \int_{0}^{T} d \tau e^{-i n \bar{\Omega} \tau} \bar{x}(\tau) \\
& =x_{L} \delta_{n 0}+\left(x_{R}-x_{L}\right) \int_{0}^{1} d u e^{-2 i n \pi u} \operatorname{sn}^{2} 2 K u,
\end{aligned}
$$

where we have changed to the $u$ variable, used the explicit trajectories (83) and again redefined the integration variable in the second line. According to 16.7 and 16.8 in Ref. [83], we have the following properties: sn $2 K(u+1)$ $=-\operatorname{sn} 2 K u, \quad$ sn $2 K(u+2 i Q)=\operatorname{sn} 2 K u, \quad$ where $Q$ $=K^{\prime} /(2 K)$ with $K^{\prime} \equiv K\left[\sqrt{1-\kappa^{2}}\right]$; and sn $2 K u$ has a simple pole at $u=i Q$, with residue $1 /(2 \kappa K)$.

To compute the Fourier coefficient (for $n \neq 0$ ) we consider the integral over the anti-clockwise contour $\Gamma$ with vertices at $0,1,2 i Q$ and $2 i Q-1$. The two oblique sides cancel each other, and

$$
\begin{aligned}
\int_{-1+2 i Q}^{2 i Q} d z e^{-2 i n \pi z} \operatorname{sn}^{2} 2 K z & =q^{4 n} \int_{-1}^{0} d s e^{-2 i n \pi z} \operatorname{sn}^{2} 2 K s \\
& =q^{-2 n} \int_{0}^{1} d u e^{-2 i n \pi u} \operatorname{sn}^{2} 2 K u,
\end{aligned}
$$

where $q=\exp \left(-\pi K^{\prime} / K\right)$. Near the pole, we have (see 16.8 and 16.3.1 in Ref. [83]) $\operatorname{sn} 2 K(u+i Q)=\operatorname{sn}\left(2 K u+i K^{\prime}\right)$ $=(\kappa \operatorname{sn} 2 K u)^{-1}=(2 \kappa K u)^{-1}\left[1+O\left(u^{2}\right)\right], \quad$ and from Cauchy's theorem the integral over the contour $\Gamma$ becomes $\left.2 \pi i\left(4 \kappa^{2} K^{2}\right)^{-1} d_{z} \exp (-2 i n \pi z)\right|_{z=i Q}=n \pi^{2} \kappa^{-2} K^{-2} q^{-n}$ which yields Eq. (84).

\section{APPENDIX E}

Here we compute the average power exchanged between the system and the environment. Let us go back to the Langevin equations (49) with the Gaussian source $\xi(t)$ described by Eq. (50). From these equations it follows that at the phase space point $(X, P)$ there is a dissipated power $w_{d}$
$=-\Gamma P$, where $\Gamma=-\int_{-\infty}^{t} d t^{\prime} H\left(t-t^{\prime}\right) X\left(t^{\prime}\right)$ and a noise power $w_{n}=\xi P$. The power dissipated in the whole ensemble is $W_{d}=-\int d X d P f(X, P) \Gamma P$.

Let us use the action-angle variables at $t=0$ as Lagrangian coordinates identifying a given trajectory. Then the power dissipated is

$$
W_{d}=-\int_{0}^{J_{s}} d J f(J) \int d \theta \Gamma P=-\int_{0}^{J_{s}} d J f(J) \Omega(J) \mathcal{D} .
$$

The average noise power in the whole ensemble is $\left\langle W_{n}\right\rangle$ $=\int_{0}^{J_{s}} d J f(J) \int d \theta\langle\xi(t) P\rangle$ where $P(\theta, J, t)$ has been perturbed away from the classical value by the action of the noise. We then get, using the Novikov trick

$$
\begin{aligned}
\langle\xi(t) P\rangle & =\int^{t} d t^{\prime} N\left(t-t^{\prime}\right) \frac{\delta P(t)}{\delta j\left(t^{\prime}\right)} \\
& =\int^{t} d t^{\prime} N\left(t-t^{\prime}\right) \frac{\partial X\left(t^{\prime}\right)}{\partial X(t)}=-\{P, N\} .
\end{aligned}
$$

We can simplify this by using the same arguments as in Appendix A:

$$
\int d \theta\{P, N\}=\int d \theta\left(\partial_{\theta} P\right)_{J}\left(\partial_{J} N\right)_{P}=\partial_{J} \int d \theta\left(\partial_{\theta} P\right)_{J} N .
$$

Performing now a last integration by parts $\int d \theta\{P, N\}$ $=\partial_{J} \int d \theta N\left(\partial_{\theta} P\right)_{J}=-\partial_{J} \int d \theta P\left(\partial_{\theta} N\right)_{J}=-\partial_{J} \mathcal{N}$.

The total average power is thus

$$
W=W_{d}+W_{n}=\int_{0}^{J_{s}} d J f(J)\left[\frac{\partial \mathcal{N}}{\partial J}-\Omega(J) \mathcal{D}\right] .
$$

Integrating by parts, knowing that $\mathcal{N}(0)=f\left(J_{s}\right)=0$, we get $W=\int_{0}^{J_{S}} d J \Omega(J) \bar{\Phi}(J)$ where $\bar{\Phi}$ is the flux. Recalling now that $\Omega=d E / d J$, with our boundary conditions we have $W$ $=E_{s} \Phi\left(E_{s}\right)+\int_{0}^{J_{s}} d J E \partial_{t} f$. We can now show that $W \sim 0$ (this is obviously true for the equilibrium solution $F_{B}$, when both terms in the expression for $W$ vanish independently). Let us write the general solution for the distribution $f$ as the following superposition $f=\int_{0}^{\infty} d r e^{-r t} c_{r} F_{r}(j)$.

Since the flux is linear in $f$, and the flux for $F_{r}$ is $K_{r}$, we get for $W$,

$$
W=\int_{0}^{\infty} d r e^{-r t} c_{r}\left(E_{s} K_{r}-r \int_{0}^{J_{s}} d J E F_{r}\right) .
$$

The integral in the second term is dominated by the upper limit (since $\sigma \sim E^{-2}$, the integral depends only logarithmically on the peak $\left.E^{*}\right)$, and $\int_{0}^{J_{s}} d J E F_{r} \sim E_{s} \int_{0}^{J_{s}} d J F_{r}$ $=E_{s} K_{r} / r$, which makes the total averaged power $W \sim 0$ as expected. 
[1] A. Guth, in The Very Early Universe, edited by G. Gibbons, S. W. Hawking, and S. T. C. Siklos (Cambridge University Press, Cambridge, England, 1983), p. 171.

[2] E. Shuryak, Phys. Rep. 61, 71 (1980).

[3] P. Shukla, A. Mohanty, S. Gupta, and M. Gleiser, Phys. Rev. C 62, 054904 (2000).

[4] G. Gamow, Z. Phys. 51, 204 (1928); R. Gurney and E. Condon, Phys. Rev. 33, 127 (1929); N. S. Krylov and V. A. Fock, Zh. Eksp. Teor. Fiz. 17, 93 (1947); V. A. Fock, Zh. Éksp. Teor. Fiz. 42, 1135 (1962) [Sov. Phys. JETP 15, 784 (1962)].

[5] L. Landau and E. Lifshitz, Quantum Mechanics (Non Relativistic Theory) (Pergamon, London, 1958).

[6] A. Migdal, Qualitative Methods in Quantum Theory (AddisonWesley, New York, 1977).

[7] H. Kramers, Physica (Amsterdam) VII, 284 (1940).

[8] J. Langer, Ann. Phys. (N.Y.) 54, 258 (1969).

[9] S. Coleman, Phys. Rev. D 15, 2929 (1977); C. Callan and S. Coleman, ibid. 16, 1762 (1977); S. Coleman, V. Glaser, and A. Martin, Commun. Math. Phys. 58, 211 (1978); S. Coleman and F. De Luccia, Phys. Rev. D 21, 3305 (1980); S. Coleman, Aspects of Symmetry (Cambridge University Press, Cambridge, England, 1985).

[10] P. Hanggi, P. Talkner, and M. Borkovec, Rev. Mod. Phys. 62, 251 (1990).

[11] I. Affleck, Phys. Rev. Lett. 46, 388 (1981); A. D. Linde, Nucl. Phys. B216, 421 (1983).

[12] J. Garriga, Phys. Rev. D 49, 5497 (1994).

[13] E. Calzetta and B. L. Hu, Phys. Rev. D 49, 6636 (1994).

[14] J. Gunton, M. San Miguel, and P. Sahni, in Phase Transitions and Critical Phenomena, edited by C. Domb and J. Lebowitz (Academic Press, London, 1983), Vol. 8, p. 267.

[15] M. Castagnino and R. Laura, Phys. Rev. A 56, 108 (1997); D. Arbo, M. Castagnino, F. Gaioli, and S. Iguri, Physica A 277, 469 (2000).

[16] J. Langer, Ann. Phys. (N.Y.) 41, 108 (1967).

[17] N. G. van Kampen, Phys. Rev. 135, A362 (1964); J. Lebowitz and O. Penrose, J. Math. Phys. 7, 98 (1966).

[18] H. Suzuki and H. Yasuta, Phys. Rev. D 57, 2500 (1998).

[19] D. Bes and J. Kurchan, The Treatment of Collective Coordinates in Many-Body Systems (World Scientific, Singapore, 1990).

[20] T. Banks, C. Bender, and T. Wu, Phys. Rev. D 8, 3346 (1973); 8, 3365 (1973).

[21] M. Gleiser, G. Marques, and R. Ramos, Phys. Rev. D 48, 1571 (1993).

[22] A. Goncharov and A. Linde, Fiz. Elm. Chastits At. Yadra 17, 837 (1986) [Sov. J. Part. Nucl. 17, 369 (1986)]; A. Linde, in Three Hundred Years of Gravitation, edited by S. Hawking and W. Israel (Cambridge University Press, Cambridge, England, 1986).

[23] V. Rubakov, Nucl. Phys. B245, 481 (1984).

[24] T. Tanaka, M. Sasaki, and K. Yamamoto, Phys. Rev. D 49, 1039 (1994); K. Yamamoto, T. Tanaka, and M. Sasaki, ibid. 51, 2968 (1995); T. Hamazaki, M. Sasaki, T. Tanaka, and K. Yamamoto, ibid. 53, 2045 (1996).

[25] L. Mersini, Phys. Rev. D 59, 123521 (1999).

[26] A. Schmid, Ann. Phys. (N.Y.) 170, 333 (1986).

[27] G. Bonini, A. Cohen, C. Rebbi, and V. Rubakov, Phys. Rev. D 60, 076004 (1999); quant-ph/9901062.
[28] J. P. Paz and S. Sinha, Phys. Rev. D 44, 1038 (1991); 45, 2823 (1992).

[29] D. Boyanovsky and C. Aragao de Carvalho, Phys. Rev. D 48, 5850 (1993); D. Boyanovsky et al., Nucl. Phys. B441, 595 (1995); B441, 609 (1995).

[30] J.-L. Gervais and B. Sakita, Phys. Rev. D 16, 3507 (1977); H. De Vega, J.-L. Gervais, and B. Sakita, Nucl. Phys. B139, 20 (1978); B142, 125 (1978).

[31] I. Affleck and F. De Luccia, Phys. Rev. D 20, 3168 (1979).

[32] U. Weiss and W. Haeffner, Phys. Rev. D 27, 2916 (1983).

[33] W. Buchmuller, Z. Fodor, T. Helbig, and D. Walliser, Ann. Phys. (N.Y.) 234, 260 (1994).

[34] K. Schleich, Phys. Rev. D 32, 1889 (1985).

[35] G. Munster, A. Strumia, and N. Tetradis, Phys. Lett. A 271, 80 (2000).

[36] V. Kiselev and K. Selivanov, Pis'ma Zh. Éksp. Teor. Fiz. 39, 72 (1984) [JETP Lett. 39, 85 (1984)].

[37] S. Weinberg, The Quantum Theory of Fields II: Modern Applications (Cambridge University Press, Cambridge, England, 1996).

[38] F. Wegner and A. Houghton, Phys. Rev. A 8, 401 (1973).

[39] A. Strumia, N. Tetradis, and C. Wetterich, Phys. Lett. B 467, 279 (1999).

[40] A. Strumia and N. Tetradis, Nucl. Phys. B542, 719 (1999); J. High Energy Phys. 11, 023 (1999).

[41] M. D'Attanasio and M. Pietroni, Nucl. Phys. B472, 711 (1996).

[42] S.-B. Liao, J. Polonyi, and D. Xu, Phys. Rev. D 51, 748 (1995); S.-B. Liao and M. Strickland, ibid. 52, 3653 (1995).

[43] D. Dalvit and F. Mazzitelli, Phys. Rev. D 54, 6338 (1996).

[44] K. Aoki, A. Horikoshi, M. Taniguchi, and H. Terao, hep-th/9812050.

[45] E. Calzetta and B.-L. Hu, Phys. Rev. D 55, 3536 (1997).

[46] H. B. Callen and T. A. Welton, Phys. Rev. 83, 34 (1951); W. Bernard and H. B. Callen, Rev. Mod. Phys. 31, 1017 (1959); M. S. Green, J. Chem. Phys. 19, 1036 (1951); R. Kubo, J. Phys. Soc. Jpn. 12, 570 (1957); Rep. Prog. Phys. 29, 255 (1966).

[47] A. O. Caldeira and A. J. Leggett, Ann. Phys. (N.Y.) 149, 374 (1983); Phys. Rev. Lett. 46, 211 (1981); A. J. Leggett et al., Rev. Mod. Phys. 59, 1 (1987).

[48] U. Weiss, Quantum Dissipative Systems (World Scientific, Singapore, 1993).

[49] A. Bray and M. Moore, Phys. Rev. Lett. 49, 1545 (1982).

[50] K. Fujikawa, Phys. Rev. E 57, 5023 (1998); K. Fujikawa and H. Terashima, ibid. 58, 7063 (1998); K. Fujikawa, S. Iso, M. Sasaki, and H. Suzuki, Phys. Rev. B 46, 10295 (1992).

[51] S. Matsumoto and M. Yoshimura, hep-ph/0008025.

[52] R. Bruninsma and P. Bak, Phys. Rev. Lett. 56, 420 (1986).

[53] W. Horsthemke and R. Lefever, Noise-induced Transitions (Springer, Berlin, 1984).

[54] M. Griffoni and P. Hanggi, Phys. Rep. 304, 229 (1998).

[55] J. Zinn-Justin, Quantum Field Theory and Critical Phenomena (Clarendon Press, Oxford, 1989).

[56] D. Hochberg, C. Molina-Paris, J. Perez-Mercader, and M. Visser, Phys. Rev. E 60, 6343 (1999); Physica A 280, 437 (2000); J. Stat. Phys. 99, 903 (2000); Phys. Lett. A 278, 177 (2001).

[57] M. Eguia and G. Mindlin, Phys. Rev. E 61, 6490 (2000).

[58] A. Starobinsky, in Field Theory, Quantum Gravity and Strings, 
edited by H. De Vega and N. Sanchez (Springer, Berlin, 1986), p. 107.

[59] E. Calzetta and B.-L. Hu, Phys. Rev. D 52, 6770 (1995).

[60] C. Kiefer, D. Polarsky, and A. Starobinsky, Int. J. Mod. Phys. D 7, 255 (1998).

[61] M. Mijic, Phys. Rev. D 42, 2469 (1990).

[62] B.-L. Hu and A. Matacz, Phys. Rev. D 51, 1577 (1995); B.-L. Hu and S. Sinha, ibid. 51, 1587 (1995); A. Campos and E. Verdaguer, ibid. 53, 1927 (1996); B.-L. Hu, Int. J. Theor. Phys. 38, 2987 (1999); R. Martín and E. Verdaguer, ibid. 38, 3049 (1999); Phys. Lett. B 465, 113 (1999); Phys. Rev. D 60, 084008 (1999); 61, 124024 (2000).

[63] E. Calzetta and E. Verdaguer, Phys. Rev. D 59, 083513 (1999).

[64] A. Vilenkin, Phys. Lett. 117B, 25 (1982); Phys. Rev. D 27, 2848 (1983); 30, 509 (1984).

[65] R. P. Feynman and F. L. Vernon, Ann. Phys. (N.Y.) 24, 118 (1963); R. P. Feynman and A. R. Hibbs, Quantum Mechanics and Path Integrals (McGraw-Hill, New York, 1965).

[66] A. Roura and E. Verdaguer, Phys. Rev. D 60, 107503 (1999).

[67] E. Calzetta, A. Roura, and E. Verdaguer, quant-ph/0011097.

[68] B. L. Hu, J. P. Paz, and Y. Zhang, Phys. Rev. D 47, 1576 (1993).

[69] H. Risken, The Fokker-Planck Equation (Springer-Verlag, Berlin, 1989).

[70] W. H. Zurek, Phys. Today 44 (10), 36 (1991); J. P. Paz, S. Habib, and W. H. Zurek, Phys. Rev. D 47, 488 (1993).

[71] M. Gell-Mann and J. B. Hartle, Phys. Rev. D 47, 3345 (1993).

[72] E. Wigner, Phys. Rev. 40, 749 (1932).
[73] J. M. Sancho and M. San Miguel, Z. Phys. B 36, 357 (1979); Z. Phys. B: Condens. Matter 43, 361 (1981).

[74] T. D. Lee and G. Wick, Phys. Rev. D 9, 2291 (1974).

[75] F. Liu and G. Mazenko, Phys. Rev. B 44, 9185 (1991); 45, 6989 (1992); 46, 5963 (1992); R. Wickham and G. Mazenko, Phys. Rev. E 55, 2300 (1997); 55, 1321 (1997); A. J. Bray, Adv. Phys. 43, 357 (1997).

[76] V. I. Arnold, Mathematical Methods of Classical Mechanics (Springer, Berlin, 1978).

[77] I. S. Gradshteyn and I. M. Ryzhik, Table of Integrals Series, and Products (Academic, New York, 1979).

[78] C. W. Gardiner, Handbook of Stochastic Methods (SpringerVerlag, Berlin, 1994).

[79] R. Courant and D. Hilbert, Methods of Mathematical Physics (Interscience, New York, 1962).

[80] J. Heading, An Introduction to Phase-Integral Methods (Methuen, London, 1962).

[81] S. Habib, Phys. Rev. D 42, 2566 (1990); M. Berry, in Chaotic Behavior of Deterministic Systems, edited by G. Iooss, R. Helleman, and R. Stora (North Holland, Amsterdam, 1983); A. Ozorio de Almeida, Hamiltonian Systems: Chaos and Quantization (Cambridge University Press, Cambridge, England, 1988).

[82] E. A. Novikov, Sov. Phys. JETP 20, 1290 (1965).

[83] Handbook of Mathematical Functions, edited by M. Abramowitz and I. Stegun (Dover, New York, 1978).

[84] E. Whittaker and G. Watson, A Course of Modern Analysis (Cambridge University Press, Cambridge, England, 1940). 